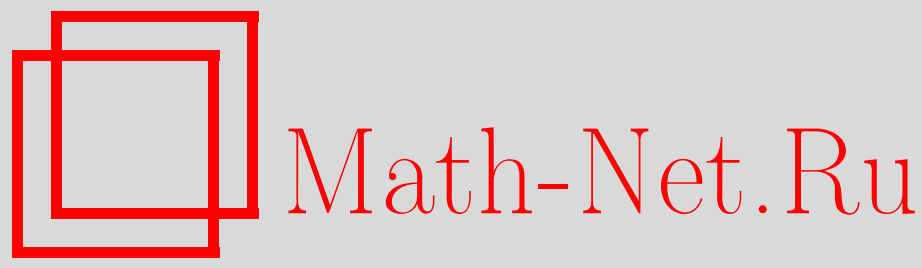

А. А. Гвоздев, Е. В. Осокина, Нейтринные процессы во внешнем магнитном поле в формализме матрицы плотности, ТМФ, 2012, том 170, номер 3, 423-447

DOI: https://doi.org/10.4213/tmf6776

Использование Общероссийского математического портала Math-Net.Ru подразумевает, что вы прочитали и согласны с пользовательским соглашением http://www.mathnet.ru/rus/agreement

Параметры загрузки :

IP : 54.80 .73 .141

26 апреля 2023 г., 14:48:56

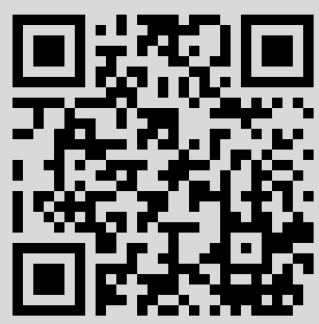




\section{НЕЙТРИННЫЕ ПРОЦЕССЫ ВО ВНЕШНЕМ МАГНИТНОМ ПОЛЕ В ФОРМАЛИЗМЕ МАТРИЦЫ ПЛОТНОСТИ}

В формализме матрицы плотности заряженной спинорной частицы, находящейся в постоянном однородном магнитном поле, развита техника вычисления скорости реакции и 4-импульса, уносимого нейтрино из плазмы, в одновершинных нейтринных процессах. С применением данной техники воспроизведены результаты для светимости в процессах нейтринного синхротронного излучения электроном (позитроном) и аннигиляции электрона и позитрона в пару нейтрино.

Ключевые слова: нейтринные процессы, магнитное поле, матрица плотности.

\section{1. ВВЕДЕНИЕ}

В настоящее время исследование нейтринных процессов в сильном магнитном поле и плотной горячей плазме - один из интенсивно развиваемых разделов космофизики. Интерес к данной тематике, в частности, связан с численным расчетом асимметричного взрыва сверхновой с коллапсом центральной части прежде всего в магниторотационной модели взрыва [1]. В этой модели напряженность магнитного поля в областях оболочки сверхновой с сильной магниторотационной неустойчивостью может достигать $B \sim 10^{16}$ Гс за типичные времена в несколько секунд [2].

Вследствие нарушения $P$-четности в процессах нейтринного излучения остатка взрыва с сильным магнитным полем формирующемуся пульсару может быть передан существенный импульс вдоль вектора напряженности магнитного поля. Различные аспекты идеи о связи асимметрии вылета нейтрино с аномально большими скоростями пульсаров, высказанной в работе [3], интенсивно развивались в последующих исследованиях [4]--[9]. В работах [10], [11] исследовался механизм генерации тороидального поля в процессах нейтринного переизлучения оболочки при магниторотационном взрыве сверхновой. Показано, что этот механизм эффективен и может привести к одностороннему взрыву сверхновой.

* Ярославский государственный университет им. П. Г. Демидова, Ярославль, Россия. E-mail: gvozdev@uniyar.ac.ru 
Другими астрофизическими объектами, у которых предполагается наличие сильного магнитного поля, являются две родственные по наблюдательным данным группы одиночных нейтронных звезд - источники мягких повторяющихся гамма-всплесков (SGR) и аномальные рентгеновские пульсары (AXP). Если считать, что основная потеря вращательного момента этих звезд происходит за счет магнитодипольного излучения, то напряженность магнитного поля на их поверхности должна составлять $B_{0} \sim 10^{14}-10^{15}$ Гс [12]. Для описания наблюдательных данных была предложена магнитарная модель [13], [14], в рамках которой исследовались рентгеновское и гамма-излучение SGR как в "спокойном" состоянии [15], так и в период гигантских вспышек [16]. В гигантских вспышках SGR за типичные времена $\Delta t \sim 100$ с излучается громадная энергия $\Delta E \sim 10^{44}-10^{46}$ эрг [12]. Предполагается, что источник такой энергии - клубок плазмы, удерживаемый сильным магнитным полем звезды [16]. Детальный анализ потерь энергии плазмы на нейтринное излучение позволяет получить ограничение на напряженность магнитного поля магнитара [17].

В условиях оболочки сверхновой с коллапсом центральной части доминирующими процессами переизлучения электронных нейтрино являются URCA-процессы [18]:

$$
\begin{aligned}
& e^{-}+p \rightleftarrows \nu_{e}+n, \\
& e^{+}+n \rightleftarrows \tilde{\nu}_{e}+p, \\
& n \rightleftarrows p+e^{-}+\tilde{\nu}_{e},
\end{aligned}
$$

последний из которых, $\beta$-распад нейтрона, кинематически подавлен. Основными процессами рождения нейтрино произвольных ароматов и их диффузии в среде оболочки являются: процесс аннигиляции электрон-позитронной пары в пару нейтрино произвольного аромата $(i=e, \mu, \tau)$ :

$$
e^{+}+e^{-} \rightarrow \nu_{i}+\tilde{\nu}_{i}
$$

упругое рассеяние нейтрино (антинейтрино) на электронах (позитронах) среды:

$$
\nu_{i}\left(\tilde{\nu}_{i}\right)+e^{\mp} \rightarrow \nu_{i}\left(\tilde{\nu}_{i}\right)+e^{\mp}
$$

упругий процесс рассеяния нейтрино (антинейтрино) на нуклонах $(N=n, p)$ :

$$
\nu_{i}\left(\tilde{\nu}_{i}\right)+N \rightarrow \nu_{i}\left(\tilde{\nu}_{i}\right)+N .
$$

Результаты детального исследования этих процессов в условиях оболочки сверхновой без магнитного поля содержатся, например, в обзоре [19]. В присутствии магнитного поля необходимо учесть не только изменение фазового объема заряженных частиц, но и модификацию $S$-матричных элементов процессов (1)-(6) с заряженными частицами, которые вследствие нарушения $P$-четности в слабых взаимодействиях содержат асимметрию по отношению к направлению магнитного поля. Кроме того, в магнитном поле становятся кинематически возможными новые нейтринные процессы, наиболее существенными из которых в условиях оболочки сверхновой являются процесс синхротронного излучения пары нейтрино $(i=e, \mu, \tau)$ :

$$
e^{\mp} \stackrel{B}{\longrightarrow} e^{\mp}+\nu_{i} \tilde{\nu}_{i}
$$


а также обратный к нему процесс рождения одиночным нейтрино (антинейтрино) электрон-позитронной пары:

$$
\nu_{i}\left(\tilde{\nu}_{i}\right) \stackrel{B}{\longrightarrow} \nu_{i}\left(\tilde{\nu}_{i}\right)+e^{+}+e^{-} .
$$

В реакциях (7) и (8) символ $B$ над стрелкой подчеркивает, что данные процессы индуцированы магнитным полем.

Отметим, что электрон-позитронная плазма, порождающая гигантскую вспышку SGR в магнитарной модели [16], прозрачна для нейтрино. Как показано в работе [17], основными процессами ее нейтринного излучения в сильном магнитном поле магнитара являются реакции (4) и (7).

Исследование указанных выше нейтринных процессов в постоянном однородном магнитном поле, а также в электромагнитных полях других конфигураций, имеет довольно продолжительную историю. В частности, вероятность $\beta$-распада нейтрона в постоянном однородном магнитном поле и угловое распределение излученного в распаде антинейтрино были впервые исследованы в работах [20], [21]. Через несколько лет эта же вероятность была независимо получена в работах [22], [23], однако распределение антинейтрино по углам вылета в этих работах не обсуждается. История развития исследования $\beta$-процессов в электромагнитных полях различных конфигураций и современное состояние теории рассмотрены в обзорной статье [24]. Наиболее общие результаты исследований URCA-процессов (1)-(3) в магнитном поле, обсуждаемых и в настоящей работе, по нашему мнению, содержатся в работах [24], [25]. В работе [25] получено релятивистское выражение для квадрата матричного элемента $\beta$-распада (пригодное для описания любых URCA-процессов) и вычислена полная вероятность процесса с учетом отдачи нуклона. Вычисление сечения захвата нейтрино релятивистским нейтроном с учетом отдачи релятивистского протона и аномальных магнитных моментов нуклонов содержится в работе [24]. Историю исследований и детальный анализ нейтрино-лептонных процессов (4) и (7) можно найти в обзорах [26], [27], а процесса (8) - в книге [28].

Наибольший интерес для астрофизики представляют такие характеристики, как скорость процесса (число переходов в единичном объеме за единицу времени):

$$
\Gamma=\frac{1}{V} \prod_{\mathrm{i}} \sum d n_{\mathrm{i}} f_{\mathrm{i}} \prod_{\mathrm{f}} \sum d n_{\mathrm{f}}\left(1-f_{\mathrm{f}}\right) \frac{\left|S_{\mathrm{if}}\right|^{2}}{\mathcal{T}},
$$

а также 4-импульс, уносимый в реакции нейтрино из единичного объема среды в единицу времени:

$$
\mathcal{P}_{\alpha}=\frac{1}{V} \prod_{\mathrm{i}} \sum d n_{\mathrm{i}} f_{\mathrm{i}} \prod_{\mathrm{f}} \sum d n_{\mathrm{f}}\left(1-f_{\mathrm{f}}\right) k_{\alpha} \frac{\left|S_{\mathrm{if}}\right|^{2}}{\mathcal{T}}
$$

Здесь $d n_{\mathrm{i}}$ и $d n_{\mathrm{f}}$ - элементы фазового объема начальных (i) и конечных (f) частиц, участвующих в реакции, $f_{\mathrm{i}}$ и $f_{\mathrm{f}}$ - их функции распределения, $\left|S_{\mathrm{if}}\right|^{2} / \mathcal{T}-$ квадрат $S$-матричного элемента процесса в единицу времени, $k_{\alpha}-4$-импульс, уносимый нейтрино в реакции, $V$ - нормировочный объем, а символ $\sum$ означает суммирование по дискретным и интегрирование по непрерывным числам заполнения частицы. Скорость процесса (9) позволяет вычислить среднее время пробега нейтрино в среде 
в нейтринопоглощающих реакциях:

$$
\bar{\tau}_{\nu}=\frac{n_{\nu}}{\Gamma},
$$

где $n_{\nu}$ - концентрация нейтрино. Нуль-компонента 4-вектора (10) определяет нейтринную светимость, а компонента вектора (10) вдоль направления магнитного поля - асимметрию в процессах переизлучения нейтрино [11]. Отметим также, что в низкоэнергетическом пределе $\left(\left|q^{2}\right| \ll m_{W}^{2}\right.$, где $q^{2}-$ квадрат переданного в реакции 4-импульса, $m_{W}$ - масса $W$-бозона), который хорошо выполняется практически во всех астрофизических приложениях, указанные выше нейтринные процессы являются одновершинными, поскольку описываются в этом пределе эффективной локальной $(V-A)$-теорией.

В настоящей работе рассмотрена техника вычисления характеристик (9) и (10) в одновершинных нейтринных процессах с использованием матрицы плотности заряженной частицы в постоянном однородном магнитном поле. Привлекательность такого способа вычисления заключается в том, что он подобен технике вычисления фейнмановских диаграмм в вакууме. Матрица плотности заряженной релятивистской частицы в постоянном однородном магнитном поле давно привлекала интерес исследователей [29]. Один из вариантов ее введения предложен в работе [30]. Заметим, что в импульсном пространстве эту матрицу естественно определить так, чтобы в бесполевом пределе при суммировании по поляризациям заряженной частицы с положительной (отрицательной) энергией получить хорошо известный результат

$$
\rho^{( \pm)}(p)=\hat{p} \pm m
$$

где $\hat{p}=p_{\mu} \gamma^{\mu}, \gamma^{\mu}$ - матрицы Дирака. Насколько нам известно, выражение для матрицы плотности, обладающей указанной асимптотикой, отсутствует в литературе.

Работа построена следующим образом. В разделе 2 получена матрица плотности заряженной частицы в постоянном однородном магнитном поле. В разделе 3 изложена техника вычисления квадрата $S$-матричного элемента одновершинных нейтринных процессов. В качестве примера приложения техники в разделе 4 вычисляется нейтринная светимость в процессе синхротронного излучения пары нейтрино. В разделе 5 суммируются полученные в работе результаты.

В работе используется система единиц $c=\hbar=k=1$.

\section{2. МАТРИЦА ПЛОТНОСТИ ЗАРЯЖЕННОЙ ЧАСТИЦЫ В ПОСТОЯННОМ ОДНОРОДНОМ МАГНИТНОМ ПОЛЕ}

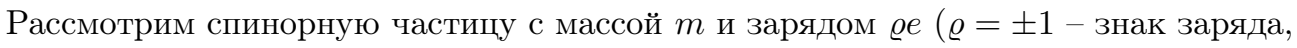
$e>0)$, находящуюся в постоянном однородном магнитном поле. Выберем ось $O z$ вдоль вектора напряженности магнитного поля $\vec{B}$, а векторный потенциал - в виде

$$
A^{\mu}=(0,0, x B, 0)
$$

Для описания спиновых свойств частицы воспользуемся проекцией оператора магнитной поляризации спина $\hat{\vec{\mu}}$ на направление магнитного поля [31]

$$
\hat{\mu}_{3}=m \Sigma_{3}+\rho_{2}[\vec{\Sigma} \times \vec{P}]_{3},
$$


где $\vec{\Sigma}$ - оператор дираковского спина, $\vec{P}=\vec{p}-\varrho e \vec{A}, \vec{p}$ - оператор кинематического импульса,

$$
\rho_{2}=\left(\begin{array}{cc}
0 & -i I \\
i I & 0
\end{array}\right)
$$

Решение уравнения Дирака с собственными значениями $s= \pm 1$ оператора $\hat{\mu}_{3}$ широко используется в литературе и может быть найдено в калибровке (12), например, в работе [24]. Такое решение с положительной энергией для частицы с произвольным знаком заряда $\varrho$ может быть записано в следующем виде:

$$
\begin{gathered}
\psi_{n, p_{2}, p_{3}, s}^{(+)}(\mathrm{x})=\frac{e^{-i\left(E_{n} t-p_{2} y-p_{3} z\right)}}{\sqrt{2 E_{n} L_{y} L_{z}}} U_{n, p_{2}, p_{3}, s}^{(+)}(\eta), \\
U_{n, p_{2}, p_{3}, s=\varrho}^{(+)}(\eta)=W_{s} \chi_{n}(\eta)-V_{-s} \chi_{n-1}(\eta), \\
U_{n, p_{2}, p_{3}, s=-\varrho}^{(+)}(\eta)=V_{-s} \chi_{n}(\eta)+W_{s} \chi_{n-1}(\eta),
\end{gathered}
$$

где $E_{n}=\sqrt{p_{3}^{2}+m^{2}+2 e B n}-$ энергия, квантовое число $n=0,1,2, \ldots$ нумерует уровни Ландау, $p_{2}$ и $p_{3}$ - проекции импульса частицы, $\mathrm{x}^{\mu}=(t, x, y, z), L_{y}$ и $L_{z}$ - нормировочные длины вдоль осей $O y$ и $O z, \eta=\sqrt{e B}\left(x-\varrho p_{2} / e B\right)$. Решение (15) выражается через нормированные функции одномерного осциллятора (функции Эрмита)

$$
\chi_{k}(\eta)=\frac{(e B)^{1 / 4} e^{-\eta^{2} / 2}}{\sqrt{2^{k} k ! \sqrt{\pi}}} H_{k}(\eta), \quad H_{k}(\eta)=(-1)^{k} e^{\eta^{2}} \frac{d^{k}}{d \eta^{k}} e^{-\eta^{2}},
$$

где $H_{k}(\eta)$ - полиномы Эрмита, и биспиноры

$$
\begin{gathered}
W_{-}=\sqrt{\frac{\tilde{p}_{\perp}+m}{2 \tilde{p}_{\perp}}}\left(\begin{array}{c}
0 \\
\sqrt{E_{n}+\tilde{p}_{\perp}} \\
0 \\
-p_{3} / \sqrt{E_{n}+\tilde{p}_{\perp}}
\end{array}\right), \quad W_{+}=\sqrt{\frac{\tilde{p}_{\perp}+m}{2 \tilde{p}_{\perp}}}\left(\begin{array}{c}
\sqrt{E_{n}+\tilde{p}_{\perp}} \\
0 \\
p_{3} / \sqrt{E_{n}+\tilde{p}_{\perp}} \\
0
\end{array}\right), \\
V_{-}=i \sqrt{\frac{0}{2 \tilde{p}_{\perp}-m}}\left(\begin{array}{c}
-p_{3} / \sqrt{E_{n}+\tilde{p}_{\perp}} \\
0 \\
\sqrt{E_{n}+\tilde{p}_{\perp}}
\end{array}\right), \quad V_{+}=i \sqrt{\frac{\tilde{p}_{\perp}-m}{2 \tilde{p}_{\perp}}}\left(\begin{array}{c}
p_{3} / \sqrt{E_{n}+\tilde{p}_{\perp}} \\
0 \\
\sqrt{E_{n}+\tilde{p}_{\perp}} \\
0
\end{array}\right) .
\end{gathered}
$$

Здесь $\tilde{p}_{\perp}=\sqrt{2 e B n+m^{2}}, p_{3}$ - компонента импульса частицы вдоль вектора напряженности магнитного поля, $m$ - масса заряженной частицы.

Решение уравнения Дирака с отрицательной энергией может быть получено из формул (15) формальной заменой $E_{n} \rightarrow-E_{n}, p_{2} \rightarrow-p_{2}, p_{3} \rightarrow-p_{3}$, что эквивалентно заменам $m \rightarrow-m$ и $V_{s} \rightarrow-V_{s}$ в биспиноре $U_{n, p_{2}, p_{3}, s}^{(+)}(\eta)$. Таким образом, отрицательно-частотное решение может быть представлено в виде

$$
\begin{gathered}
\psi_{n, p_{2}, p_{3}, s}^{(-)}(\mathrm{x})=\frac{e^{i\left(E_{n} t-p_{2} y-p_{3} z\right)}}{\sqrt{2 E_{n} L_{y} L_{z}}} U_{n, p_{2}, p_{3}, s}^{(-)}(\tilde{\eta}), \\
U_{n, p_{2}, p_{3}, s=\varrho}^{(-)}(\tilde{\eta})=\widetilde{W}_{s} \chi_{n}(\tilde{\eta})+\widetilde{V}_{-s} \chi_{n-1}(\tilde{\eta}), \\
U_{n, p_{2}, p_{3}, s=-\varrho}^{(-)}(\tilde{\eta})=-\widetilde{V}_{-s} \chi_{n}(\tilde{\eta})+\widetilde{W}_{s} \chi_{n-1}(\tilde{\eta}),
\end{gathered}
$$


где $\widetilde{W}_{s}=W_{s}(m \rightarrow-m), \widetilde{V}_{s}=V_{s}(m \rightarrow-m), \tilde{\eta}=\sqrt{e B}\left(x+\varrho p_{2} / e B\right)$ и подразумевается, что знак заряда $\varrho$ для решения с отрицательной энергией тот же, что и для решения с положительной энергией.

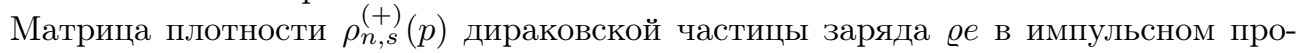
странстве, соответствующая решению (15) уравнения Дирака с положительной энергией и собственным значением $s$ оператора (13), определяется из соотношения

$$
\psi_{n, p_{2}, p_{3}, s}^{(+)}(\mathrm{x}) \bar{\psi}_{n, p_{2}, p_{3}, s}^{(+)}\left(\mathrm{x}^{\prime}\right)=\frac{e^{i \varrho \Phi\left(\mathrm{x}, \mathrm{x}^{\prime}\right)}}{2 E_{n} L_{y} L_{z}} \int_{-\infty}^{\infty} e^{-i p\left(\mathrm{x}-\mathrm{x}^{\prime}\right)} \rho_{n, s}^{(+)}(p) \frac{d p_{1}}{2 \pi},
$$

где $p\left(\mathrm{x}-\mathrm{x}^{\prime}\right)=p_{\mu}\left(\mathrm{x}-\mathrm{x}^{\prime}\right)^{\mu}, p^{\mu}=\left(E_{n}, p_{1}, p_{2}, p_{3}\right)$ может интерпретироваться как 4-импульс частицы, и трансляционно-неинвариантная фаза

$$
\Phi\left(\mathrm{x}, \mathrm{x}^{\prime}\right)=\frac{e B}{2}\left(x+x^{\prime}\right)\left(y-y^{\prime}\right) .
$$

Матрицу плотности $\rho_{n, s}^{(+)}(p)$ можно записать в виде

$$
\begin{aligned}
\rho_{n, s=\rho}^{(+)}(p)= & (-1)^{n} e^{-u / 2}\left\{\left[\left(1+\frac{m}{\tilde{p}_{\perp}}\right) \hat{p}_{\|}+\tilde{p}_{\perp}+m\right] \Pi_{\varrho} L_{n}(u)-\right. \\
& \left.-\left[\left(1-\frac{m}{\tilde{p}_{\perp}}\right) \hat{p}_{\|}-\tilde{p}_{\perp}+m\right] \Pi_{-\varrho} L_{n-1}(u)+2\left[\hat{p}_{\perp}-i \varrho \frac{\hat{p}_{\|}}{\tilde{p}_{\perp}}(p \varphi \gamma)\right] L_{n-1}^{1}(u)\right\}, \\
\rho_{n, s=-\rho}^{(+)}(p)= & (-1)^{n} e^{-u / 2}\left\{\left[\left(1-\frac{m}{\tilde{p}_{\perp}}\right) \hat{p}_{\|}-\tilde{p}_{\perp}+m\right] \Pi_{\varrho} L_{n}(u)-\right. \\
& \left.-\left[\left(1+\frac{m}{\tilde{p}_{\perp}}\right) \hat{p}_{\|}+\tilde{p}_{\perp}+m\right] \Pi_{-\varrho} L_{n-1}(u)+2\left[\hat{p}_{\perp}+i \varrho \frac{\hat{p}_{\|}}{\tilde{p}_{\perp}}(p \varphi \gamma)\right] L_{n-1}^{1}(u)\right\} .
\end{aligned}
$$

Здесь $\hat{p}_{\|}=(p \tilde{\Lambda} \gamma)=E_{n} \gamma_{0}-p_{3} \gamma_{3}, \hat{p}_{\perp}=(p \Lambda \gamma)=p_{1} \gamma_{1}+p_{2} \gamma_{2},(p \varphi \gamma)=p_{2} \gamma_{1}-p_{1} \gamma_{2}$, $\varphi_{\mu \nu}=F_{\mu \nu} / B$ и $\tilde{\varphi}_{\mu \nu}=\widetilde{F}_{\mu \nu} / B$ - безразмерные тензор и дуальный тензор электромагнитного поля, $\Lambda_{\mu \nu}=(\varphi \varphi)_{\mu \nu}, \tilde{\Lambda}_{\mu \nu}=(\tilde{\varphi} \tilde{\varphi})_{\mu \nu}, \Pi_{\varrho}-$ оператор проекции спина частицы на направление магнитного поля (см. формулу (90) в приложении А). Отметим, что входящие в выражения (22) и (23) структуры $\hat{p}_{\|}, \hat{p}_{\perp},(p \varphi \gamma), \tilde{p}_{\perp}, \Pi_{\varrho}$, а также аргумент полиномов Лагерра $u=2 p_{\perp}^{2} / e B=2\left(p_{1}^{2}+p_{2}^{2}\right) / e B$ являются инвариантами относительно преобразований Лоренца вдоль вектора напряженности магнитного поля. Эффективное разбиение 4-мерного пространства в постоянном однородном магнитном поле на два ортогональных подпространства - параллельное (\|) и перпендикулярное $(\perp)$, а также алгебра матриц Дирака в этих подпространствах приведены в приложении А. Подробный вывод формул (22) и (23) содержится в приложении Б.

После суммировании по поляризациям $s$ матрица плотности приводится к виду

$$
\begin{aligned}
\rho_{n}^{(+)}(p)= & \sum_{s= \pm \rho} \rho_{n, s}^{(+)}(p)=(-1)^{n} 2 e^{-u / 2} \times \\
& \times\left[\left(\hat{p}_{\|}+m\right)\left(\Pi_{\varrho} L_{n}(u)-\Pi_{-\varrho} L_{n-1}(u)\right)+2 \hat{p}_{\perp} L_{n-1}^{1}(u)\right] .
\end{aligned}
$$

Отметим, что выражение для $\rho_{n}^{(+)}(p)$ может быть получено при использовании любого из проектирующих операторов, обсуждаемых подробно в книге [31], поскольку 
не содержит информации о спиновых свойствах заряженной дираковской частицы. При "наивном" суммировании матрицы плотности (24) по $n$, т. е. в предположении, что в пределе слабого поля $(B \rightarrow 0)$ дискретный спектр энергий заряженной частицы переходит в непрерывный $\left(E_{n} \rightarrow E=\sqrt{p^{2}+m^{2}}\right)$, получаем стандартное вакуумное выражение:

$$
\begin{aligned}
\sum_{n=0}^{\infty} \rho_{n}^{(+)}(p)=2 e^{-u / 2}\left(\hat{p}_{\|}+m\right) \sum_{n=0}^{\infty}(-1)^{n}\left[\Pi_{\varrho} L_{n}(u)-\Pi_{-\varrho} L_{n-1}(u)\right]+ \\
\quad+4 e^{-u / 2} \hat{p}_{\perp} \sum_{n=1}^{\infty}(-1)^{n} L_{n-1}^{1}(u)=\hat{p}_{\|}+m-\hat{p}_{\perp}=\hat{p}+m
\end{aligned}
$$

где было использовано правило суммирования обобщенных полиномов Лагерра [32]:

$$
\sum_{n=0}^{\infty}(-1)^{n} \frac{(n+k) !}{n ! k !} L_{n+k}^{m}(2 x)=\frac{e^{x}}{2^{k+m+1}} L_{k}^{m}(x)
$$

при значениях $k=0$ и $m=0,1$.

В случае нерелятивистской частицы, пренебрегая всеми поперечными к полю компонентами импульса и полагая $\hat{p}_{\|}=m \hat{v}_{\|}$, из формул $(22)$ и $(23)$ получим

$$
\begin{aligned}
\rho_{n, s=\varrho}^{(+)}(p) & =(-1)^{n} 2 e^{-u / 2} L_{n}(u) m\left(1+\hat{v}_{\|}\right) \Pi_{\varrho}, \\
\rho_{n, s=-\varrho}^{(+)}(p) & =(-1)^{n+1} 2 e^{-u / 2} L_{n-1}(u) m\left(1+\hat{v}_{\|}\right) \Pi_{-\varrho},
\end{aligned}
$$

где $v^{\mu}=(1,0,0, \mathrm{v}) / \sqrt{1-\mathrm{v}^{2}}-4$-скорость движения среды вдоль направления поля. Нетрудно убедиться, что матрица плотности (27) описывает состояние с определенной проекцией оператора дираковского спина $\Sigma_{3}$ на направление магнитного поля.

Поскольку волновая функция нерелятивистской заряженной частицы с аномальным магнитным моментом, например протона, не зависит от аномального момента, приведенные выражения описывают матрицу плотности с определенной поляризацией также и в этом случае. Отметим, что учет взаимодействия аномального магнитного момента с магнитным полем снимает вырождение энергии по уровням Ландау:

$$
E_{n, s}=m+\frac{p_{3}^{2}}{2 m}+\frac{e B n}{m}-\tilde{g} \varrho \frac{e B s}{2 m},
$$

где число $n$ нумерует уровни Ландау, $\tilde{g}$ - аномальный магнитный момент в ядерных магнетонах для нуклонов и магнетонах Бора для электронов.

Просуммированная по $s$ матрица плотности, соответствующая решению уравнения Дирака с отрицательной энергией (19), получается из соотношения (20) формальной заменой $p^{\mu} \rightarrow-p^{\mu}$, что приводит к выражению

$$
\begin{gathered}
\sum_{s= \pm 1} \psi_{n, p_{2}, p_{3}, s}^{(-)}(\mathrm{x}) \bar{\psi}_{n, p_{2}, p_{3}, s}^{(-)}\left(\mathrm{x}^{\prime}\right)=\frac{e^{-i \varrho \Phi\left(\mathrm{x}, \mathrm{x}^{\prime}\right)}}{2 E_{n} L_{y} L_{z}} \int_{-\infty}^{\infty} e^{i p\left(\mathrm{x}-\mathrm{x}^{\prime}\right)} \rho_{n}^{(-)}(p) \frac{d p_{1}}{2 \pi}, \\
\rho_{n}^{(-)}(p)=(-1)^{n} 2 e^{-u / 2}\left\{\left(\hat{p}_{\|}-m\right)\left[\Pi_{\varrho} L_{n}(u)-\Pi_{-\varrho} L_{n-1}(u)\right]+2 \hat{p}_{\perp} L_{n-1}^{1}(u)\right\} .
\end{gathered}
$$

Здесь знак заряда $\varrho$ такой же, как и для положительно-частотного решения. 
Для полноты изложения приведем известные матрицы плотности для безмассового нейтрино левой спиральности с 4-импульсом $k^{\mu}=(\omega, \vec{k})$ :

$$
\psi_{k}^{(\nu)}(\mathrm{x}) \bar{\psi}_{k}^{(\nu)}\left(\mathrm{x}^{\prime}\right)=\frac{e^{-i k\left(\mathrm{x}-\mathrm{x}^{\prime}\right)}}{2 \omega V} \rho^{(\nu)}(k), \quad \rho^{(\nu)}(k)=\frac{1}{2} \hat{k}\left(1-\gamma_{5}\right),
$$

и для электронейтральной частицы с массой $m_{N}$ и 4-импульсом $P^{\mu}=(E, \vec{P})$ :

$$
\sum_{s= \pm 1} \psi_{P, s}^{(N)}(\mathrm{x}) \bar{\psi}_{P, s}^{(N)}\left(\mathrm{x}^{\prime}\right)=\frac{e^{-i P\left(\mathrm{x}-\mathrm{x}^{\prime}\right)}}{2 E V} \rho^{(N)}(P), \quad \rho^{(N)}(P)=\widehat{P}+m_{N},
$$

где $\gamma_{5}=-i \gamma_{0} \gamma_{1} \gamma_{2} \gamma_{3}$ и $V=L_{x} L_{y} L_{z}-$ нормировочный объем. В нерелятивистском пределе матрица плотности поляризованной электронейтральной частицы имеет вид

$$
\rho_{s}^{(N)}(P)=m_{N}\left(1+\hat{v}_{\|}\right) \Pi_{s},
$$

не меняющийся и при учете магнитного момента частицы, например нейтрона. Однако в этом случае энергия частицы явно зависит от ее поляризации и определяется выражением

$$
E_{s}=m_{N}+\frac{\vec{P}^{2}}{2 m_{N}}-g \frac{e B s}{2 m_{N}},
$$

где $g$ - магнитный момент нейтрона в ядерных магнетонах.

\section{3. СЛАБЫЕ ОДНОВЕРШИННЫЕ ПРОЦЕССЫ}

В этом разделе мы покажем, как работает формализм матрицы плотности, на следующих примерах: процессы рассеяния нейтрино на электронах (5) и нуклонах (6), а также URCA-процесс (1). $S$-матричные элементы и их квадраты для кроссинг-симметричных процессов могут быть получены соответствующими заменами 4-импульсов частиц.

В низкоэнергетическом пределе, когда переданные в реакции энергия и импульс много меньше массы $W$-бозона $\left(m_{W} \simeq 80\right.$ ГэВ [33]), локальный эффективный лагранжиан процессов (5) и (6) может быть записан единообразно:

$$
\mathcal{L}_{\mathrm{eff}}^{(1)}(\mathrm{x})=\frac{G_{\mathrm{F}}}{\sqrt{2}}\left[\bar{\psi}^{(Q)}(\mathrm{x}) \gamma_{\alpha}\left(c_{\mathrm{v}}+c_{\mathrm{a}} \gamma_{5}\right) \psi^{(Q)}(\mathrm{x})\right]\left[\bar{\psi}^{(\nu)}(\mathrm{x}) \gamma_{\alpha}\left(1+\gamma_{5}\right) \psi^{(\nu)}(\mathrm{x})\right]
$$

где $G_{\mathrm{F}}$ - константа Ферми, $\psi^{(Q)}(\mathrm{x})$ - оператор электрона (нуклона), $\psi^{(\nu)}(\mathrm{x})$ - оператор нейтринного поля, $c_{\mathrm{v}}$ и $c_{\mathrm{a}}$ - векторные и аксиальные константы эффективных нейтральных слабых токов. Отметим, что в рассматриваемом пределе значения этих констант для процесса (5) зависят от аромата нейтрино [28]:

$$
\begin{aligned}
& c_{\mathrm{v}}^{(e)}=+\frac{1}{2}+2 \sin ^{2} \theta_{\mathrm{W}}, \quad c_{\mathrm{a}}^{(e)}=+\frac{1}{2}, \quad \nu=\nu_{e}, \\
& c_{\mathrm{v}}^{(x)}=-\frac{1}{2}+2 \sin ^{2} \theta_{\mathrm{W}}, \quad c_{\mathrm{a}}^{(x)}=-\frac{1}{2}, \quad \nu_{x}=\nu_{\mu}, \nu_{\tau},
\end{aligned}
$$

где $\theta_{\mathrm{W}}-$ угол Вайнберга $\left(\sin ^{2} \theta_{\mathrm{W}} \simeq 0.23\right.$ [33]), тогда как для процессов вида (6) константы слабых токов зависят от типа нуклона [34]:

$$
\begin{aligned}
& c_{\mathrm{v}}^{(p)}=\frac{0.07}{2}, \quad c_{\mathrm{a}}^{(p)}=\frac{1.09}{2}, \quad Q=p(\text { протон }), \\
& c_{\mathrm{v}}^{(N)}=-\frac{1}{2}, \quad c_{\mathrm{a}}^{(N)}=-\frac{0.91}{2}, \quad Q=N(\text { нейтрон) } .
\end{aligned}
$$


В низкоэнергетическом пределе локальный эффективный лагранжиан URCAпроцесса (1) может быть представлен в виде [26]

$$
\mathcal{L}_{\mathrm{eff}}^{(2)}(\mathrm{x})=\frac{G_{\mathrm{F}} \cos \theta_{\mathrm{C}}}{\sqrt{2}}\left[\bar{\psi}^{(N)}(\mathrm{x}) \gamma_{\alpha}\left(g_{\mathrm{v}}+g_{\mathrm{a}} \gamma_{5}\right) \psi^{(p)}(\mathrm{x})\right]\left[\bar{\psi}^{(\nu)}(\mathrm{x}) \gamma_{\alpha}\left(1+\gamma_{5}\right) \psi^{(e)}(\mathrm{x})\right]
$$

где $\theta_{\mathrm{C}}-$ угол Кабиббо $\left(\sin \theta_{\mathrm{C}} \simeq 0.22[33]\right), \psi^{(N)}(\mathrm{x}), \psi^{(p)}(\mathrm{x}), \psi^{(e)}(\mathrm{x}), \psi^{(\nu)}(\mathrm{x})-$ операторы нейтронного, протонного, электронного, нейтринного полей соответственно, $g_{\mathrm{v}} \simeq 1$ и $g_{\mathrm{a}} \simeq 1.26$ - векторная и аксиальная константы заряженного нуклонного тока. $S$-матричные элементы процессов (5) и $(6)$, составленные по локальному эффективному лагранжиану (34), могут быть записаны в виде

$$
\begin{gathered}
S_{\mathrm{if}}^{(1)}=\frac{i G_{1}}{\sqrt{2}} \int\left[\bar{\psi}_{n^{\prime}, p_{2}^{\prime}, p_{3}^{\prime}, s^{\prime}}^{(Q)}(\mathrm{x}) \widetilde{O}_{\alpha}(c) \psi_{n, p_{2}, p_{3}, s}^{(Q)}(\mathrm{x})\right]\left[\bar{\psi}_{k^{\prime}}^{(\nu)}(\mathrm{x}) O_{\alpha} \psi_{k}^{(\nu)}(\mathrm{x})\right] d^{4} \mathrm{x} \\
\widetilde{O}_{\alpha}(c)=\gamma_{\alpha}\left(1+c \gamma_{5}\right), \quad O_{\alpha}=\gamma_{\alpha}\left(1+\gamma_{5}\right)
\end{gathered}
$$

где $G_{1}=G_{\mathrm{F}} c_{\mathrm{v}}, c=c_{\mathrm{a}} / c_{\mathrm{v}}, \psi_{n, p_{2}, p_{3}, s}^{(Q)}(\mathrm{x}), \psi_{n^{\prime}, p_{2}^{\prime}, p_{3}^{\prime}, s^{\prime}}^{(\mathrm{x})}, \psi_{k}^{(\nu)}(\mathrm{x}), \psi_{k^{\prime}}^{(\nu)}(\mathrm{x})-$ волновые функции нуклона и нейтрино в начальном и конечном состояниях и интегрирование ведется по 4-мерному нормировочному объему $\Omega=\mathcal{T} L_{x} L_{y} L_{z}$.

$S$-матричный элемент процесса $(1)$, соответствующий локальному эффективному лагранжиану (37), запишется в виде

$$
S_{\mathrm{if}}^{(2)}=\frac{i G_{2}}{\sqrt{2}} \int\left[\bar{\psi}_{P^{\prime}, s^{\prime}}^{(N)}(\mathrm{x}) \widetilde{O}_{\alpha}(g) \psi_{m, P_{2}, P_{3}, s}^{(p)}(\mathrm{x})\right]\left[\bar{\psi}_{q^{\prime}}^{(\nu)}(\mathrm{x}) O_{\alpha} \psi_{m^{\prime}, q_{2}, q_{3}, s^{\prime \prime}}^{(e)}(\mathrm{x})\right] d^{4} \mathrm{x},
$$

где $G_{2}=G_{\mathrm{F}} g_{\mathrm{v}} \cos \theta_{\mathrm{C}}, g=g_{\mathrm{a}} / g_{\mathrm{v}} \simeq 1.26, \psi_{m, P_{2}, P_{3}, s}^{(p)}(\mathrm{x})$ и $\psi_{m^{\prime}, q_{2}, q_{3}, s^{\prime \prime}}^{(\mathrm{e})}(\mathrm{x})-$ волновые функции протона и электрона, $\psi_{P^{\prime}, s^{\prime}}^{(N)}(\mathrm{x})$ - нейтрона, $\psi_{q^{\prime}}^{(\nu)}(\mathrm{x})$ - нейтрино.

При использовании формализма матрицы плотности квадраты $S$-матричных элементов, просуммированные по поляризациям частиц, представляются в виде

$$
\begin{aligned}
\sum_{s, s^{\prime}= \pm 1}\left|S_{\mathrm{if}}^{(1)}\right|^{2}= & \frac{G_{1}^{2}}{2} \int \frac{d p_{1} d p_{1}^{\prime}}{4 \pi^{2}} \operatorname{Sp}\left[\rho_{n^{\prime}}^{(Q)}\left(p^{\prime}\right) \widetilde{O}_{\alpha}(c) \rho_{n}^{(Q)}(p) \widetilde{O}_{\beta}(c)\right] \times \\
& \times \operatorname{Sp}\left[\rho^{(\nu)}\left(k^{\prime}\right) O_{\alpha} \rho^{(\nu)}(k) O_{\beta}\right] \int d^{4} \mathrm{x} d^{4} \mathrm{x}^{\prime} \frac{e^{-i\left(p+k-p^{\prime}-k^{\prime}\right)\left(\mathrm{x}-\mathrm{x}^{\prime}\right)}}{16 \varepsilon_{n} \omega \varepsilon_{n^{\prime}}^{\prime} \omega^{\prime} L_{y}^{2} L_{z}^{2} V^{2}}
\end{aligned}
$$

где $p^{(\prime) \mu}=\left(\varepsilon_{n^{(\prime)}}^{(\prime)}, \vec{p}^{(\prime)}\right), k^{(\prime) \mu}=\left(\omega^{(\prime)}, \vec{k}^{(\prime)}\right)$ - 4-импульсы заряженных частиц и нейтрино в начальном (конечном) состоянии,

$$
\begin{aligned}
\sum_{s, s^{\prime}, s^{\prime \prime}= \pm 1}\left|S_{\mathrm{if}}^{(2)}\right|^{2}= & \frac{G_{2}^{2}}{2} \int \frac{d q_{1} d P_{1}}{4 \pi^{2}} \operatorname{Sp}\left[\rho^{(N)}\left(P^{\prime}\right) \widetilde{O}_{\alpha}(g) \rho_{m}^{(p)}(P) \widetilde{O}_{\beta}(g)\right] \times \\
& \times \operatorname{Sp}\left[\rho^{(\nu)}\left(q^{\prime}\right) O_{\alpha} \rho_{m^{\prime}}^{(e)}(q) O_{\beta}\right] \int d^{4} \mathrm{x} d^{4} \mathrm{x}^{\prime} \frac{e^{-i\left(P+q-P^{\prime}-q^{\prime}\right)\left(\mathrm{x}-\mathrm{x}^{\prime}\right)}}{16 E_{m} \varepsilon_{m^{\prime}} E^{\prime} q_{0}^{\prime} L_{y}^{2} L_{z}^{2} V^{2}}
\end{aligned}
$$

где $P^{\mu}=\left(E_{m}, \vec{P}\right), q^{\mu}=\left(\varepsilon_{m^{\prime}}, \vec{q}\right), P^{\prime \mu}=\left(E^{\prime}, \vec{P}^{\prime}\right)$ и $q^{\prime \mu}=\left(q_{0}^{\prime}, \vec{q}^{\prime}\right)$ - 4-импульсы протона, электрона, нейтрона и нейтрино. 
Интегрирование квадратов S-матричных элементов по х и $\mathrm{x}^{\prime}$ тривиально и приводит к следующим результатам:

$$
\begin{gathered}
\sum_{s, s^{\prime}= \pm 1}\left|S_{\mathrm{if}}^{(1)}\right|^{2}=\frac{(-1)^{n+n^{\prime}} \pi^{2} G_{1}^{2} \mathcal{T}}{2 \varepsilon_{n} \omega \varepsilon_{n^{\prime}}^{\prime} \omega^{\prime} L_{y}^{2} L_{z}^{2} V} \int\left[L_{\alpha \beta}^{(Q)} L_{\alpha \beta}^{(\nu)}\right] e^{-\left(u+u^{\prime}\right) / 2} \delta^{(4)}\left(p+k-p^{\prime}-k^{\prime}\right) d p_{1} d p_{1}^{\prime} \\
L_{\alpha \beta}^{(Q)}=(-1)^{n+n^{\prime}} e^{\left(u+u^{\prime}\right) / 2} \frac{1}{4} \operatorname{Sp}\left[\rho_{n^{\prime}}^{(Q)}\left(p^{\prime}\right) \widetilde{O}_{\alpha}(c) \rho_{n}^{(Q)}(p) \widetilde{O}_{\beta}(c)\right] \\
L_{\alpha \beta}^{(\nu)}=\operatorname{Sp}\left[\rho^{(\nu)}\left(k^{\prime}\right) O_{\alpha} \rho^{(\nu)}(k) O_{\beta}\right] \\
\sum_{s, s^{\prime}, s^{\prime \prime}= \pm 1}\left|S_{\mathrm{if}}^{(2)}\right|^{2}=\frac{(-1)^{m+m^{\prime}} \pi^{2} G_{2}^{2} \mathcal{T}}{2 E_{m} \varepsilon_{m^{\prime}} E^{\prime} q_{0}^{\prime} L_{y}^{2} L_{z}^{2} V} \int\left[N_{\alpha \beta} L_{\alpha \beta}\right] e^{-\left(v+v^{\prime}\right) / 2} \delta^{(4)}\left(P+q-P^{\prime}-q^{\prime}\right) d q_{1} d P_{1} \\
N_{\alpha \beta}=(-1)^{m} e^{v / 2} \frac{1}{2} \operatorname{Sp}\left[\rho^{(N)}\left(P^{\prime}\right) \widetilde{O}_{\alpha}(g) \rho_{m}^{(p)}(P) \widetilde{O}_{\beta}(g)\right] \\
L_{\alpha \beta}=(-1)^{m^{\prime}} e^{v^{\prime} / 2} \frac{1}{2} \operatorname{Sp}\left[\rho^{(\nu)}\left(q^{\prime}\right) O_{\alpha} \rho_{m^{\prime}}^{(e)}(q) O_{\beta}\right]
\end{gathered}
$$

где $u=2 p_{\perp}^{2} / e B, u^{\prime}=2 p_{\perp}^{\prime 2} / e B, v=2 P_{\perp}^{2} /(e B), v^{\prime}=2 q_{\perp}^{2} / e B$.

При подстановке выражения $(43)$ в $\mathcal{P}_{\mu}(10)$ получим явно ковариантное выражение

$$
\begin{aligned}
\mathcal{P}_{\mu}^{(1)}= & \frac{G_{1}^{2}}{8(2 \pi)^{8}} \sum_{n, n^{\prime}=0}^{\infty}(-1)^{n+n^{\prime}} \int \frac{d^{3} k}{\omega} f_{\nu}(\omega) \int \frac{d^{3} k^{\prime}}{\omega^{\prime}}\left[1-f_{\nu}\left(\omega^{\prime}\right)\right]\left(k^{\prime}-k\right)_{\mu} \times \\
& \times \int \frac{d^{3} p}{\varepsilon_{n}} f_{Q}\left(\varepsilon_{n}\right) \int \frac{d^{3} p^{\prime}}{\varepsilon_{n^{\prime}}^{\prime}}\left[1-f_{Q}\left(\varepsilon_{n^{\prime}}^{\prime}\right)\right] \delta^{(4)}\left(p+k-p^{\prime}-k^{\prime}\right) e^{-\left(u+u^{\prime}\right) / 2}\left[L_{\alpha \beta}^{(Q)} L_{\alpha \beta}^{(\nu)}\right],
\end{aligned}
$$

где $f_{\nu}\left(\omega^{(\prime)}\right)$ и $f_{Q}\left(\varepsilon_{n^{(\prime)}}^{(\prime)}\right)$ - функции распределения начальных (конечных) нейтрино и заряженной частицы.

Аналогичным образом $\mathcal{P}_{\mu}$ в реакции (1) принимает вид

$$
\begin{aligned}
\mathcal{P}_{\mu}^{(2)}= & \frac{G_{2}^{2}}{8(2 \pi)^{8}} \sum_{m, m^{\prime}=0}^{\infty}(-1)^{m+m^{\prime}} \int \frac{d^{3} q^{\prime}}{q_{0}^{\prime}}\left[1-f_{\nu}\left(q_{0}^{\prime}\right)\right] q_{\mu}^{\prime} \int \frac{d^{3} P}{E_{m}} f_{p}\left(E_{m}\right) \times \\
& \times \int \frac{d^{3} q}{\varepsilon_{m^{\prime}}} f_{e}\left(\varepsilon_{m^{\prime}}\right) \int \frac{d^{3} P^{\prime}}{E^{\prime}}\left[1-f_{N}\left(E^{\prime}\right)\right] \delta^{(4)}\left(P+q-P^{\prime}-q^{\prime}\right) e^{-\left(v+v^{\prime}\right) / 2}\left[N_{\alpha \beta} L_{\alpha \beta}\right] .
\end{aligned}
$$

Поскольку шпур от нечетного числа $\gamma$-матриц равен нулю, то после небольших преобразований с использованием коммутационных свойств матрицы $\gamma_{5}$ с проекци- 
онным оператором $\left(\Pi_{\varrho} \gamma_{5}=\gamma_{5} \Pi_{\varrho}\right)$ нетрудно получить следующие выражения:

$$
\begin{aligned}
& L_{\alpha \beta}^{(Q)}= \operatorname{Sp}\left[\left\{\hat{p}_{\|}^{\prime}\left(\Pi_{\varrho} L_{n^{\prime}}\left(u^{\prime}\right)-\Pi_{-\varrho} L_{n^{\prime}-1}\left(u^{\prime}\right)\right)+2 \hat{p}_{\perp}^{\prime} L_{n^{\prime}-1}^{1}\left(u^{\prime}\right)\right\} \gamma_{\alpha} \times\right. \\
&\left.\times\left\{\hat{p}_{\|}\left(\Pi_{\varrho} L_{n}(u)-\Pi_{-\varrho} L_{n-1}(u)\right)+2 \hat{p}_{\perp} L_{n-1}^{1}(u)\right\} \gamma_{\beta}\left(1+c^{2}+2 c \gamma_{5}\right)\right]+ \\
&+ m_{Q}^{2}\left(1-c^{2}\right) \operatorname{Sp}\left[\left(\Pi_{\varrho} L_{n^{\prime}}\left(u^{\prime}\right)-\Pi_{-\varrho} L_{n^{\prime}-1}\left(u^{\prime}\right)\right) \gamma_{\alpha}\left(\Pi_{\varrho} L_{n}(u)-\Pi_{-\varrho} L_{n-1}(u)\right) \gamma_{\beta}\right], \\
& L_{\alpha \beta}^{(\nu)}=2 \operatorname{Sp}\left[\hat{k}^{\prime} \gamma_{\alpha} \hat{k} \gamma_{\beta}\left(1+\gamma_{5}\right)\right]
\end{aligned}
$$

для шпуров, входящих в формулу (45), и

$$
\begin{aligned}
N_{\alpha \beta}= & \operatorname{Sp}\left[\widehat{P}^{\prime} \gamma_{\alpha}\left\{\widehat{P}_{\|}\left(\Pi_{+} L_{m}(v)-\Pi_{-} L_{m-1}(v)\right)+2 \widehat{P}_{\perp} L_{m-1}^{1}(v)\right\} \gamma_{\beta}\left(1+g^{2}+2 g \gamma_{5}\right)\right]+ \\
& +m_{N} m_{p}\left(1-g^{2}\right) \operatorname{Sp}\left[\gamma_{\alpha}\left(\Pi_{+} L_{m}(v)-\Pi_{-} L_{m-1}(v)\right) \gamma_{\beta}\right] \\
L_{\alpha \beta}= & 2 \operatorname{Sp}\left[\hat{q}^{\prime} \gamma_{\alpha}\left\{\hat{q}_{\|}\left(\Pi_{-} L_{m^{\prime}}\left(v^{\prime}\right)-\Pi_{+} L_{m^{\prime}-1}\left(v^{\prime}\right)\right)+2 \hat{q}_{\perp} L_{m^{\prime}-1}^{1}\left(v^{\prime}\right)\right\} \gamma_{\beta}\left(1+\gamma_{5}\right)\right]
\end{aligned}
$$

для шпуров, входящих в формулу (46).

При использовании свойств (91)-(94), (98) и (100) (см. приложение А) приведенные выше громоздкие шпуры вычисляются без особых трудностей. Техника вычисления интегралов, входящих в $\mathcal{P}_{\mu}^{(1)}(45)$ и $\mathcal{P}_{\mu}^{(2)}(46)$, по компонентам импульсов заряженных частиц, поперечных вектору напряженности магнитного поля, подробно изложена в приложении В.

\section{4. СВЕТИМОСТЬ В ПРОЦЕССЕ НЕЙТРИННОГО СИНХРОТРОННОГО ИЗЛУЧЕНИЯ}

В настоящем разделе в формализме матрицы плотности вычисляется нейтринная светимость в процессе синхротронного излучения нейтринной пары электроном (позитроном). История изучения этого процесса насчитывает более сорока лет [35]. Ссылки на ранние работы, а также анализ современного состояния его исследования содержатся в обзорах [26], [27]. Выражение для нейтринной светимости процесса и интерполяционная формула для численного расчета получены в работе [36], где предполагалось, что плазма прозрачна для родившихся нейтрино.

Вычислим $\mathcal{P}_{\mu}^{(1)}(10)$ для нейтрино определенного аромата в том же предположении, что и в работе [36]. Результат вычислений необходимо просуммировать по всем ароматам нейтрино $i=e, \mu, \tau$, учитывая значения векторных и аксиальных констант (35) слабых токов. При переходе от канала рассеяния к нейтринному синхротронному излучению необходимо сделать замену 4-импульса нейтрино $k_{\mu} \rightarrow-k_{\mu}$, и выражение (45) приводится к виду

$$
\begin{aligned}
\mathcal{P}_{\mu}= & \frac{G_{1}^{2}}{8(2 \pi)^{8}} \int \frac{d^{3} k}{\omega} \int \frac{d^{3} k^{\prime}}{\omega^{\prime}}\left(k+k^{\prime}\right)_{\mu} L_{\alpha \beta}^{(\nu)} \sum_{n, n^{\prime}=0}^{\infty}(-1)^{n+n^{\prime}} \times \\
& \times \int \frac{d^{3} p}{\varepsilon_{n}} f\left(\varepsilon_{n}\right) \int \frac{d^{3} p^{\prime}}{\varepsilon_{n^{\prime}}^{\prime}}\left[1-f\left(\varepsilon_{n^{\prime}}^{\prime}\right)\right] e^{-\left(u+u^{\prime}\right) / 2} L_{\alpha \beta}^{(e)} \delta^{(4)}\left(p-p^{\prime}-k-k^{\prime}\right) .
\end{aligned}
$$

Напомним, что здесь $p^{(\prime) \mu}=\left(\varepsilon_{n^{(\prime)}}^{(\prime)}, \vec{p}^{(\prime)}\right)$ - 4-векторы импульса начального (конечного) электрона, электронный шпур $L_{\alpha \beta}^{(e)}$ соответствует выражению $(47)$ при $\varrho=-1$, 
нейтринный шпур $L_{\alpha \beta}^{(\nu)}$ - выражению (48), и для электронов используются равновесные функции распределения

$$
f\left(\varepsilon_{n}\right)=\frac{1}{e^{\varepsilon_{n} / T-\eta}+1},
$$

где $\eta=\mu / T$. Выражение (51) может быть ковариантно проинтегрировано по импульсам нейтрино. Введем тензорный интеграл $I_{\alpha \beta}$, который довольно легко вычисляется:

$$
I_{\alpha \beta}=\int \frac{d^{3} k}{\omega} \int \frac{d^{3} k^{\prime}}{\omega^{\prime}} \delta^{(4)}\left(k+k^{\prime}-q\right) L_{\alpha \beta}^{(\nu)}=\frac{16 \pi}{3}\left(q_{\alpha} q_{\beta}-q^{2} g_{\alpha \beta}\right) \theta\left(q^{2}\right) .
$$

Для дальнейших вычислений удобно ввести интегральную единицу

$$
\int d^{4} q \delta^{(4)}\left(p-p^{\prime}-q\right)=1
$$

тогда выражение (51) может быть приведено к виду:

$$
\begin{aligned}
\mathcal{P}_{\mu}= & \frac{G_{1}^{2}}{3(2 \pi)^{7}} \int d^{4} q q_{\mu} \theta\left(q^{2}\right) \sum_{n, n^{\prime}=0}^{\infty}(-1)^{n+n^{\prime}} \int \frac{d^{3} p}{\varepsilon_{n}} f\left(\varepsilon_{n}\right) \int \frac{d^{3} p^{\prime}}{\varepsilon_{n^{\prime}}^{\prime}}\left[1-f\left(\varepsilon_{n^{\prime}}^{\prime}\right)\right] \times \\
& \times e^{-\left(u+u^{\prime}\right) / 2} \delta^{(4)}\left(p-p^{\prime}-q\right)\left(q_{\alpha} q_{\beta} L_{\alpha \beta}^{(e)}-q^{2} g_{\alpha \beta} L_{\alpha \beta}^{(e)}\right) .
\end{aligned}
$$

Рассмотрим нулевую компоненту $Q_{\mathrm{S}}$ этого 4-вектора (нейтринную светимость). При вычислении свертки $L_{\alpha \beta}^{(e)}$ с векторами $q_{\alpha}$ и $q_{\beta}$ в светимости не следует учитывать члены, линейные по $c$, поскольку они линейны либо по $p_{3}$, либо по $p_{3}^{\prime}$ и зануляются при интегрировании по этим переменным. В результате получим

$$
\begin{aligned}
q_{\alpha} q_{\beta} L_{\alpha \beta}^{(e)}= & 2\left(1+c^{2}\right)\left\{-\left(p \tilde{\Lambda} p^{\prime}\right) q_{\perp}^{2}\left[L_{n}(u) L_{n^{\prime}-1}\left(u^{\prime}\right)+L_{n-1}(u) L_{n^{\prime}}\left(u^{\prime}\right)\right]+\right. \\
& +\left(2(p \tilde{\Lambda} q)\left(p^{\prime} \tilde{\Lambda} q\right)-q_{\|}^{2}\left(p^{\prime} \tilde{\Lambda} p\right)\right)\left[L_{n}(u) L_{n^{\prime}}\left(u^{\prime}\right)+L_{n-1}(u) L_{n^{\prime}-1}\left(u^{\prime}\right)\right]+ \\
& +4(p \Lambda q)\left(p^{\prime} \tilde{\Lambda} q\right) L_{n-1}^{1}(u)\left[L_{n^{\prime}}\left(u^{\prime}\right)-L_{n^{\prime}-1}\left(u^{\prime}\right)\right]+ \\
& +4\left(p^{\prime} \Lambda q\right)(p \tilde{\Lambda} q) L_{n^{\prime}-1}^{1}\left(u^{\prime}\right)\left[L_{n}(u)-L_{n-1}(u)\right]+ \\
& \left.+8\left(2(p \Lambda q)\left(p^{\prime} \Lambda q\right)+q^{2}\left(p^{\prime} \Lambda p\right)\right) L_{n-1}^{1}(u) L_{n^{\prime}-1}^{1}\left(u^{\prime}\right)\right\}+ \\
& +2 m^{2}\left(1-c^{2}\right)\left\{q_{\|}^{2}\left[L_{n}(u) L_{n^{\prime}}\left(u^{\prime}\right)+L_{n-1}(u) L_{n^{\prime}-1}\left(u^{\prime}\right)\right]+\right. \\
& \left.+q_{\perp}^{2}\left[L_{n}(u) L_{n^{\prime}-1}\left(u^{\prime}\right)+L_{n-1}(u) L_{n^{\prime}}\left(u^{\prime}\right)\right]\right\},
\end{aligned}
$$

где $u=2 p_{\perp}^{2} / e B, u^{\prime}=2 p_{\perp}^{\prime 2} / e B, n^{(\prime)}-$ уровни Ландау начальной (конечной) частицы.

Заметим, что вследствие сохранения векторного тока выполняется тождество

$$
\left.q_{\alpha} q_{\beta} L_{\alpha \beta}^{(e)}\right|_{c=0}=0,
$$

так что бо́льшая часть членов в выражении (56) равна нулю.

Свертка $g_{\alpha \beta} L_{\alpha \beta}^{(e)}$ имеет простой компактный вид:

$$
\begin{aligned}
g_{\alpha \beta} L_{\alpha \beta}^{(e)}= & -4 m^{2}\left(1-c^{2}\right)\left[L_{n}(u)-L_{n-1}(u)\right]\left[L_{n^{\prime}}(u)-L_{n^{\prime}-1}\left(u^{\prime}\right)\right]+4\left(1+c^{2}\right) \times \\
& \times\left\{\left(p^{\prime} \tilde{\Lambda} p\right)\left[L_{n^{\prime}}\left(u^{\prime}\right) L_{n-1}(u)+L_{n^{\prime}-1}\left(u^{\prime}\right) L_{n}(u)\right]+\right. \\
& \left.+8\left(p^{\prime} \Lambda p\right) L_{n-1}^{1}(u) L_{n^{\prime}-1}^{1}\left(u^{\prime}\right)\right\} .
\end{aligned}
$$


Далее приведем результаты вычисления содержащихся в формуле (55) интегралов по поперечным к полю компонентам импульсов электронов в терминах нормированных функций Лагерра [31], [37]:

$$
F_{n^{\prime}, n}(v)=\sqrt{\frac{n^{\prime} !}{n !}} v^{\left(n-n^{\prime}\right) / 2} e^{-v / 2} L_{n^{\prime}}^{n-n^{\prime}}(v)=n^{\prime} ! I_{n, n^{\prime}}(v) .
$$

Скалярный, векторные и тензорный интегралы (см. приложение В) в терминах этих функций могут быть представлены в виде

$$
\begin{aligned}
S^{\left(n^{\prime}, n\right)}(v)= & \int d^{2} p_{\perp} \int d^{2} p_{\perp}^{\prime} \delta^{(2)}\left(\vec{p}_{\perp}-\vec{p}_{\perp}^{\prime}-\vec{q}_{\perp}\right) L_{n}(u) L_{n^{\prime}}\left(u^{\prime}\right) e^{-\left(u+u^{\prime}\right) / 2}= \\
= & (-1)^{n^{\prime}-n} \frac{\pi e B}{2} F_{n^{\prime}, n}^{2}(v), \\
V_{\alpha}^{\left(n^{\prime}, n\right)}(v)= & \int d^{2} p_{\perp} \int d^{2} p_{\perp}^{\prime} \delta^{(2)}\left(\vec{p}_{\perp}-\vec{p}_{\perp}^{\prime}-\vec{q}_{\perp}\right) p_{\perp \alpha} L_{n-1}^{1}(u) L_{n^{\prime}}\left(u^{\prime}\right) e^{-\left(u+u^{\prime}\right) / 2}= \\
= & (-1)^{n^{\prime}-n-1} \frac{\pi e B}{4} \sqrt{\frac{n}{v}} q_{\perp \alpha} F_{n^{\prime}, n}(v) F_{n^{\prime}, n-1}(v), \\
V_{\alpha}^{\left(n, n^{\prime}\right)}(v)= & \int d^{2} p_{\perp} \int d^{2} p_{\perp}^{\prime} \delta^{(2)}\left(\vec{p}_{\perp}-\vec{p}_{\perp}^{\prime}-\vec{q}_{\perp}\right) p_{\perp \alpha}^{\prime} L_{n}(u) L_{n^{\prime}-1}^{1}\left(u^{\prime}\right) e^{-\left(u+u^{\prime}\right) / 2}= \\
= & (-1)^{n-n^{\prime}-1} \frac{\pi e B}{4} \sqrt{\frac{n^{\prime}}{v}} q_{\perp \alpha} F_{n^{\prime}, n}(v) F_{n^{\prime}-1, n}(v), \\
T_{\alpha \beta}^{\left(n, n^{\prime}\right)}(v)= & \int d^{2} p_{\perp} \int d^{2} p_{\perp}^{\prime} \delta^{(2)}\left(\vec{p}_{\perp}-\vec{p}_{\perp}^{\prime}-\vec{q}_{\perp}\right) p_{\perp \alpha} p_{\perp \beta}^{\prime} L_{n-1}^{1}(u) L_{n^{\prime}-1}^{1}\left(u^{\prime}\right) e^{-\left(u+u^{\prime}\right) / 2}= \\
& (-1)^{n^{\prime}-n} \frac{\pi e B}{16} \frac{\sqrt{n n^{\prime}}}{v}\left[\left(2 q_{\perp \alpha} q_{\perp \beta}-q_{\perp}^{2} \Lambda_{\alpha \beta}\right) F_{n^{\prime}, n-1}(v) F_{n^{\prime}-1, n}(v)+\right. \\
& \left.+q_{\perp}^{2} \Lambda_{\alpha \beta} F_{n^{\prime}, n}(v) F_{n^{\prime}-1, n-1}(v)\right],
\end{aligned}
$$

где $v=q_{\perp}^{2} /(2 e B)$.

После вычисления интегралов по поперечным к полю импульсам электронов нейтринная светимость процесса может быть приведена к виду

$$
\begin{aligned}
Q_{\mathrm{S}}= & \frac{G_{1}^{2} e B}{6(2 \pi)^{6}} \int d^{4} q q_{0} \theta\left(q^{2}\right) \sum_{n, n^{\prime}} \int_{-\infty}^{\infty} \frac{d p_{3}}{\varepsilon_{n}} \int_{-\infty}^{\infty} \frac{d p_{3}^{\prime}}{\varepsilon_{n^{\prime}}^{\prime}} f\left(\varepsilon_{n}\right)\left[1-f\left(\varepsilon_{n^{\prime}}^{\prime}\right)\right] \delta\left(\varepsilon_{n}-\varepsilon_{n^{\prime}}^{\prime}-q_{0}\right) \times \\
& \times \delta\left(p_{3}-p_{3}^{\prime}-q_{3}\right)\left\{\left(1+c^{2}\right) q^{2}\left[2 e B\left(n+n^{\prime}\right)(\Psi(v)-\Phi(v))-q^{2} \Psi(v)\right]-\right. \\
& \left.-2 m^{2}\left[q^{2}\left(\Phi(v)-2 c^{2} \Psi(v)\right)+c^{2} q_{\perp}^{2}(\Phi(v)-\Psi(v))\right]\right\}
\end{aligned}
$$

где $\Phi(v)=F_{n^{\prime}, n}^{2}(v)+F_{n^{\prime}-1, n-1}^{2}(v)$ и $\Psi(v)=F_{n^{\prime}, n-1}^{2}(v)+F_{n^{\prime}-1, n}^{2}(v)$.

Полученное выражение совпадает с соответствующим результатом работы [36].

Нейтринная светимость в процессе аннигиляции (4) может быть легко получена из формулы (64) при заменах в подинтегральном выражении $\varepsilon_{n^{\prime}}^{\prime} \rightarrow-\varepsilon_{n^{\prime}}^{\prime}, p_{3}^{\prime} \rightarrow-p_{3}^{\prime}$, $1-f\left(\varepsilon_{n^{\prime}}^{\prime}\right) \rightarrow f\left(\varepsilon_{n^{\prime}}^{\prime}\right)$ с заменой знака химического потенциала $\mu$ в функции распределения $f\left(\varepsilon_{n^{\prime}}^{\prime}\right)$ и у члена в фигурных скобках, пропорционального квадрату массы электрона. Последняя замена обусловлена использованием матрицы плотности $\rho_{n^{\prime}}^{(-)}\left(p^{\prime}\right)(29)$ для позитрона $(\varrho=-1)$, которая отличается от $\rho_{n^{\prime}}^{(+)}\left(p^{\prime}\right)(24)$ для электрона $(\varrho=-1)$ знаком перед массой частицы. 
В случае сильного магнитного поля концентрация электронов и позитронов на уровнях Ландау с $n \geqslant 1$ экспоненциально подавлена, поэтому в процессе (4) ограничимся рассмотрением вкладов либо с $n=0$, либо с $n^{\prime}=0$, а в процессе $(7)$ - вклада с $n^{\prime}=0$. Тогда выражение для нейтринной светимости (64) существенно упрощается:

$$
\begin{aligned}
\sum_{n=0}^{\infty} Q_{\mathrm{S}}^{(n, 0)}= & \frac{G_{1}^{2} e B}{6(2 \pi)^{6}} \sum_{n=0}^{\infty} \int d^{4} q q_{0} \theta\left(q^{2}\right) I_{n}\left(q_{0}, q_{3}, \eta, T\right)\left\{\left(1+c^{2}\right) q^{2}\left(2 e B n-q_{\|}^{2}\right) F_{0, n-1}^{2}(v)-\right. \\
& \left.-2 m^{2}\left[\left(q_{\|}^{2}-\left(1-c^{2}\right) q_{\perp}^{2}\right) F_{0, n}^{2}(v)-c^{2}\left(2 q_{\|}^{2}-q_{\perp}^{2}\right) F_{0, n-1}^{2}(v)\right]\right\}
\end{aligned}
$$

где было использовано известное соотношение для функций Лагерра [37]: $F_{0, n}^{2}(v)=$ $v F_{0, n-1}^{2}(v) / n$. В выражении $(65)$ под $I_{n}\left(q_{0}, q_{3}, \eta, T\right)$ понимается интеграл

$$
I_{n}\left(q_{0}, q_{3}, \eta, T\right)=\int_{-\infty}^{\infty} \frac{d p_{3}}{\varepsilon_{n}} \int_{-\infty}^{\infty} \frac{d p_{3}^{\prime}}{\varepsilon_{0}^{\prime}} \delta\left(\varepsilon_{n}-\varepsilon_{0}^{\prime}-q_{0}\right) \delta\left(p_{3}-p_{3}^{\prime}-q_{3}\right) f\left(\varepsilon_{n}\right)\left[1-f\left(\varepsilon_{0}^{\prime}\right)\right] .
$$

Для ультрарелятивистской плазмы $\left(\varepsilon_{n}^{2}, \varepsilon_{0}^{\prime 2} \gg m^{2}\right)$ этот интеграл легко вычисляется:

$$
I_{n}\left(q_{0}, q_{3}, \eta, T\right)=\frac{2}{2 e B n-q_{\|}^{2}} \theta\left(2 e B n-q_{\|}^{2}\right) \Phi_{n}\left(q_{0}, q_{3}, \eta, T\right),
$$

где

$$
\begin{aligned}
\Phi_{n}\left(q_{0}, q_{3}, \eta, T\right)= & {\left[\exp \left(\frac{q_{0}}{2 T} \frac{q_{\|}^{2}+2 e B n}{q_{\|}^{2}}-\frac{q_{3}}{2 T} \frac{q_{\|}^{2}-2 e B n}{q_{\|}^{2}}-\eta\right)+1\right]^{-1} \times } \\
& \times\left[\exp \left(\frac{q_{0}+q_{3}}{2 T} \frac{q_{\|}^{2}-2 e B n}{q_{\|}^{2}}+\eta\right)+1\right]^{-1}+\left(q_{3} \rightarrow-q_{3}\right) .
\end{aligned}
$$

Таким образом, нейтринная светимость горячей плазмы в синхротроне (7) при переходе электрона на основной уровень Ландау описывается выражением

$$
\begin{aligned}
\sum_{n=0}^{\infty} Q_{S}^{(n, 0)}= & \frac{G_{1}^{2} e B}{6(2 \pi)^{5}}\left(1+c^{2}\right) \sum_{n=0}^{\infty} \int_{0}^{\infty} d q_{0} q_{0} \int_{-\infty}^{\infty} d q_{3} \times \\
& \times \int_{0}^{\infty} d q_{\perp}^{2} \theta\left(q^{2}\right) \theta\left(2 e B n-q_{\|}^{2}\right) q^{2} F_{0, n-1}^{2}(v) \Phi_{n}\left(q_{0}, q_{3}, \eta, T\right) .
\end{aligned}
$$

Легко видеть, что $Q_{\mathrm{S}}^{(n, 0)}$ обращается в нуль при $n=0$.

Приведем далее выражение для нейтринной светимости в кроссинг-процессе аннигиляции (4):

$$
\begin{aligned}
\sum_{n=0}^{\infty} Q_{\mathrm{A}}^{(n, 0)}= & \frac{G_{1}^{2} e B}{6(2 \pi)^{5}} \sum_{n=0}^{\infty} \int_{0}^{\infty} d q_{0} q_{0} \int_{-\infty}^{\infty} d q_{3} \times \\
& \times \int_{0}^{\infty} d q_{\perp}^{2} \theta\left(q^{2}\right) \theta\left(q_{\|}^{2}-2 e B n\right) \mathcal{F}_{n}\left(q_{\|}^{2}, q_{\perp}^{2}\right) \Phi_{n}\left(q_{0}, q_{3}, \eta, T\right),
\end{aligned}
$$


где

$$
\begin{aligned}
\mathcal{F}_{n}\left(q_{\|}^{2}, q_{\perp}^{2}\right)= & \left(1+c^{2}\right) q^{2} F_{0, n-1}^{2}(v)+\frac{2 m^{2}}{q_{\|}^{2}-2 e B n} \times \\
& \times\left[\left(q_{\|}^{2}-\left(1-c^{2}\right) q_{\perp}^{2}\right) F_{0, n}^{2}(v)-c^{2}\left(2 q_{\|}^{2}-q_{\perp}^{2}\right) F_{0, n-1}^{2}(v)\right] .
\end{aligned}
$$

В $\mathcal{F}_{n}\left(q_{\|}^{2}, q_{\perp}^{2}\right)$ намеренно удержан член, пропорциональный квадрату массы электрона $m^{2}$, поскольку в отличие от синхротрона светимость в процессе аннигиляции не зануляется даже в случае, когда электрон и позитрон находятся на основном уровне Ландау.

При $n=0$ интегрирование $Q_{\mathrm{A}}^{(0,0)}$ по $q_{\perp}^{2}$ тривиально:

$$
Q_{\mathrm{A}}^{(0,0)}=\frac{G_{1}^{2} e B m^{2}}{6(2 \pi)^{5}}\left(1+c^{2}\right) \int_{0}^{\infty} d q_{0} q_{0} \int_{-\infty}^{\infty} d q_{3} q_{\|}^{2} \theta\left(q_{\|}^{2}\right) \Phi_{0}\left(q_{0}, q_{3}, \eta, T\right) .
$$

В асимптотике сверхсильного магнитного поля $\left(n=n^{\prime}=0\right)$ полученная нейтринная светимость в процессе аннигиляции ультрарелятивистской пары линейна по полю и пропорциональна квадрату массы электрона.

При $\eta=0$ функция $\Phi_{0}\left(q_{0}, q_{3}, \eta, T\right)$ упрощается, и двукратный интеграл $(72)$ вычисляется аналитически:

$$
Q_{\mathrm{A}}^{(B)}=\frac{\zeta(3)}{48 \pi^{3}}\left(1+c^{2}\right) G_{1}^{2} e B m^{2} T^{5} .
$$

Этот результат совпадает с выражением для нейтринной светимости ультрарелятивистской невырожденной плазмы в асимптотике сверхсильного магнитного поля, полученным в работе [38].

Для сравнения оценим вклад в светимость первого уровня Ландау $(n=1)$, используя следующие безразмерные отношения:

$$
R_{\mathrm{S}}^{(1)}(T, B)=\frac{Q_{S}^{(1,0)}}{Q_{\mathrm{A}}^{(B)}},
$$

где $Q_{\mathrm{S}}^{(1,0)}$ - суммарная по электронам и позитронам светимость при синхротронном переходе с уровня $n=1$ на уровень $n^{\prime}=0$ и

$$
R_{\mathrm{A}}^{(1)}(T, B)=\frac{Q_{\mathrm{A}}^{(0,0)}+Q_{\mathrm{A}}^{(1,0)}+Q_{\mathrm{A}}^{(0,1)}}{Q_{\mathrm{A}}^{(B)}} .
$$

Эти отношения показывают, насколько нейтринные светимости ультрарелятивистской невырожденной плазмы в процессах (7) и (4) отличаются от асимптотического выражения (73). Нетрудно привести формулы (74) и (75) к виду

$$
\begin{aligned}
& R_{\mathrm{S}}^{(1)}=\frac{64}{\pi^{2} \zeta(3)}\left(\frac{T}{m}\right)^{2} I_{\mathrm{S}}\left(\sqrt{\frac{e B}{2 T^{2}}}\right), \\
& R_{\mathrm{A}}^{(1)}=1+\frac{64}{\pi^{2} \zeta(3)}\left(\frac{T}{m}\right)^{2} I_{\mathrm{A}}\left(\sqrt{\frac{e B}{2 T^{2}}}\right),
\end{aligned}
$$


где введены следующие функции:

$$
\begin{aligned}
& I_{\mathrm{S}}(\alpha)=\alpha^{7} \int_{-\infty}^{\infty} d v \int_{0}^{1} d u\left[e^{-u}+u-1\right] \Phi_{1}(u, v ; \alpha), \\
& I_{\mathrm{A}}(\alpha)=\alpha^{7} \int_{-\infty}^{\infty} d v \int_{1}^{\infty} d u\left[e^{-u}+u-1\right] \Phi_{1}(u, v ; \alpha), \\
& \Phi_{1}(u, v ; \alpha)=\left\{\exp \left[\frac{\alpha}{u}\left((1+u) \sqrt{u+v^{2}}-(u-1) v\right)\right]+1\right\}^{-1} \times \\
& \times\left\{\exp \left[\frac{\alpha}{u}(u-1)\left(\sqrt{u+v^{2}}+v\right)\right]+1\right\}^{-1} .
\end{aligned}
$$

Отметим, что в пределе сильного магнитного поля выражения (76) и (77) хорошо согласуются с интерполяционными формулами, полученными при $\eta=0$ для процесса (7) в работе [39] и процесса (4) - в работе [38].

Полученный результат может быть использован для оценки нейтринных потерь из приповерхностной области магнитара, заполненной электрон-позитронной плазмой, в период гигантской вспышки SGR [16]. Для характерных значений температуры $T \gtrsim 1$ МэВ и напряженности магнитного поля $10^{15} \lesssim B \lesssim 10^{16}$ Гс этой плазмы отношения (76) и (77) составляют десятки [17]. Отсюда следует, что в период вспышечной активности магнитара потери энергии плазмы за счет нейтрино велики и не оставляют необходимого энергетического запаса на радиационное излучение. Таким образом, в рамках магнитарной модели трудно объяснить энергетику гамма-излучения гигантской вспышки SGR.

\section{5. ЗАКЛЮЧЕНИЕ}

В настоящей работе получено инвариантное импульсное представление для матрицы плотности заряженной релятивистской спинорной частицы, находящейся в постоянном однородном магнитном поле. В формализме матрицы плотности развита техника вычисления скорости реакции и 4-импульса, уносимого нейтрино из плазмы, в одновершинных нейтринных процессах. Эффективность этой техники продемонстрирована на примере вычисления нейтринных светимостей в процессах аннигиляции электрона и позитрона в пару нейтрино и нейтринного синхротронного излучения электроном (позитроном).

ПРИЛОЖЕНИЕ А

\section{Алгебра $\gamma$-матриц Дирака во внешнем магнитном поле}

Для любой частицы с импульсом $p_{\mu}$, находящейся в электромагнитном поле, можно ввести удобный для анализа квантовых процессов с ее участием базис. Заметим, что конфигурация чисто магнитного поля, наиболее важная в приложении к астрофизическим объектам, обладает набором специфических свойств, использование которых существенно упрощает расчеты конкретных реакций.

Из электродинамики известно, что электромагнитное поле полностью определяется тензором напряженностей $F_{\mu \nu}$. В дополнение к нему также вводится дуально сопряженный тензор $\widetilde{F}_{\mu \nu}=\varepsilon_{\mu \nu \rho \sigma} F^{\rho \sigma} / 2$. Выберем систему координат таким образом, 
чтобы ось $O z$ была направлена вдоль вектора напряженности постоянного однородного магнитного поля $\vec{B}=(0,0, B)$. В такой системе отсчета тензоры $F_{\mu \nu}$ и $\widetilde{F}_{\mu \nu}$ имеют следующий вид:

$$
F_{\mu \nu}=B\left(\begin{array}{cccc}
0 & 0 & 0 & 0 \\
0 & 0 & -1 & 0 \\
0 & +1 & 0 & 0 \\
0 & 0 & 0 & 0
\end{array}\right), \quad \widetilde{F}_{\mu \nu}=B\left(\begin{array}{cccc}
0 & 0 & 0 & +1 \\
0 & 0 & 0 & 0 \\
0 & 0 & 0 & 0 \\
-1 & 0 & 0 & 0
\end{array}\right) .
$$

В дальнейшем удобно пользоваться не самим тензором электромагнитного поля и дуальным к нему тензором, а их безразмерными аналогами

$$
\varphi_{\mu \nu}=\frac{F_{\mu \nu}}{B}, \quad \tilde{\varphi}_{\mu \nu}=\frac{\widetilde{F}_{\mu \nu}}{B},
$$

явный вид которых в выбранной нами системе отсчета представлен числовыми матрицами в формуле (80). Также удобно использовать коварианты, составленные из этих тензоров и 4-вектора импульса заряженной частицы, в параллельном $(0,3)$ и перпендикулярном $(1,2)$ подпространствах. Значительный вклад в развитие техники вычислений в случае сильного магнитного поля сделали Скобелев и Лоскутов, построившие так называемую "двумерную электродинамику" [40], [41].

Представляет интерес анализ алгебры введенных безразмерных тензоров (81). Начнем с бинарных произведений

$$
\Lambda_{\mu \nu}=(\varphi \varphi)_{\mu \nu}=\varphi_{\mu \rho} \varphi^{\rho}{ }_{\nu}, \quad \tilde{\Lambda}_{\mu \nu}=(\tilde{\varphi} \tilde{\varphi})_{\mu \nu}=\tilde{\varphi}_{\mu \rho} \tilde{\varphi}^{\rho}{ }_{\nu} .
$$

В отличие от антисимметричных тензоров $\varphi_{\mu \nu}$ и $\tilde{\varphi}_{\mu \nu}$ тензоры $\Lambda_{\mu \nu}$ и $\tilde{\Lambda}_{\mu \nu}$ симметричны в соответствии с общими свойствами сверток тензоров. В выбранной нами системе координат эти тензоры имеют следующий явный вид:

$$
\tilde{\Lambda}_{\mu \nu}=\left(\begin{array}{cccc}
1 & 0 & 0 & 0 \\
0 & 0 & 0 & 0 \\
0 & 0 & 0 & 0 \\
0 & 0 & 0 & -1
\end{array}\right), \quad \Lambda_{\mu \nu}=\left(\begin{array}{cccc}
0 & 0 & 0 & 0 \\
0 & 1 & 0 & 0 \\
0 & 0 & 1 & 0 \\
0 & 0 & 0 & 0
\end{array}\right)
$$

Из явного представления тензоров видно, что они не являются линейно независимыми, а связаны друг с другом посредством метрического тензора $g_{\mu \nu}$ :

$$
\tilde{\Lambda}_{\mu \nu}-\Lambda_{\mu \nu}=g_{\mu \nu}
$$

Проведенный анализ показывает, что наличие внешнего постоянного однородного магнитного поля естественным образом разбивает четырехмерное пространство Минковского на два непересекающихся подпространства: двумерное евклидово подпространство с метрическим тензором $\Lambda_{\mu \nu}$, ортогональное вектору напряженности магнитного поля $\vec{B}$, и двумерное псевдоевклидово подпространство с метрическим тензором $\tilde{\Lambda}_{\mu \nu}$. Безразмерные тензоры электромагнитного поля $\varphi_{\mu \nu}$ и $\tilde{\varphi}_{\mu \nu}$ играют роль тензоров Леви-Чивита (полностью антисимметричных тензоров) этих подпространств и обладают следующими свойствами:

$$
\begin{aligned}
& \tilde{\varphi}_{\mu \nu} \tilde{\varphi}_{\rho \sigma}=\tilde{\Lambda}_{\mu \sigma} \tilde{\Lambda}_{\nu \rho}-\tilde{\Lambda}_{\mu \rho} \tilde{\Lambda}_{\nu \sigma} \\
& \varphi_{\mu \nu} \varphi_{\rho \sigma}=\Lambda_{\mu \rho} \Lambda_{\nu \sigma}-\Lambda_{\mu \sigma} \Lambda_{\nu \rho} .
\end{aligned}
$$


Для введенного набора тензоров справедливы бинарные соотношения

$$
\begin{aligned}
& (\tilde{\varphi} \varphi)_{\mu \nu}=(\tilde{\varphi} \Lambda)_{\mu \nu}=(\tilde{\Lambda} \varphi)_{\mu \nu}=(\tilde{\Lambda} \Lambda)_{\mu \nu}=0, \\
& (\tilde{\Lambda} \tilde{\Lambda})_{\mu \nu}=\tilde{\Lambda}_{\mu \nu}, \quad(\Lambda \Lambda)_{\mu \nu}=-\Lambda_{\mu \nu}, \\
& (\tilde{\Lambda} \tilde{\varphi})_{\mu \nu}=\tilde{\varphi}_{\mu \nu}, \quad(\Lambda \varphi)_{\mu \nu}=-\varphi_{\mu \nu} .
\end{aligned}
$$

При проведении вычислений оказывается удобным ввести специальные обозначения для каждого из подпространств: $\perp$ - для евклидова подпространства с метрикой $\Lambda_{\mu \nu}$ и $\|$ - для псевдоевклидова подпространства с метрикой $\tilde{\Lambda}_{\mu \nu}$. При таком соглашении произвольный 4-вектор $A^{\mu}=\left(A_{0}, A_{1}, A_{2}, A_{3}\right)$ можно разбить на две ортогональные составляющие:

$$
A_{\mu}=\tilde{\Lambda}_{\mu \nu} A^{\nu}-\Lambda_{\mu \nu} A^{\nu}=A_{\| \mu}-A_{\perp \mu},
$$

где $A_{\|}^{\mu}=\left(A_{0}, 0,0, A_{3}\right)$ и $A_{\perp}^{\mu}=\left(0, A_{1}, A_{2}, 0\right)$ в соответствии со свойством (84). Такое разбиение позволяет ввести скалярное произведение векторов в каждом подпространстве по отдельности:

$$
\begin{gathered}
(A B)=(A B)_{\|}-(A B)_{\perp}, \\
(A B)_{\|}=(A \tilde{\Lambda} B)=A^{\mu} \tilde{\Lambda}_{\mu \nu} B^{\nu}, \\
(A B)_{\perp}=(A \Lambda B)=A^{\mu} \Lambda_{\mu \nu} B^{\nu},
\end{gathered}
$$

где $A_{\mu}$ и $B_{\mu}-$ произвольные 4-векторы.

Деление четырехмерного пространства на два непересекающихся подпространства приводит к эффективной модификации свойств $\gamma$-матриц. Будем обозначать $\gamma$-матрицы $\|$-подпространства как $\gamma_{\|}^{\mu}=\tilde{\Lambda}^{\mu \nu} \gamma_{\nu}$, а $\perp$-подпространства - как $\gamma_{\perp}^{\mu}=$ $\Lambda^{\mu \nu} \gamma_{\nu}$

Введем проекционные операторы фермиона $\Pi_{\sigma}$ :

$$
\Pi_{\sigma}=\frac{1}{2}\left[1-\frac{i \sigma}{2}(\gamma \varphi \gamma)\right]=\frac{1}{2}\left[1+\sigma i \gamma_{1} \gamma_{2}\right],
$$

где учтен явный вид тензора $\varphi_{\mu \nu}(81)$ в выбранной системе отсчета. Соответственно $\sigma=+1$ отвечает фермионному состоянию со спином, направленным по магнитному полю, а $\sigma=-1$ - состоянию со спином, направленным против магнитного поля. Отметим следующие мультипликативные и аддитивные свойства проекционных операторов:

$$
\Pi_{\sigma} \Pi_{\sigma}=\Pi_{\sigma}, \quad \Pi_{\sigma} \Pi_{-\sigma}=0, \quad \Pi_{\sigma}+\Pi_{-\sigma}=1,
$$

а также их коммутационные свойства по отношению к $\gamma$-матрицам:

$$
\Pi_{\sigma} \gamma_{\|}^{\mu}=\gamma_{\|}^{\mu} \Pi_{\sigma}, \quad \Pi_{\sigma} \gamma_{\perp}^{\mu}=\gamma_{\perp}^{\mu} \Pi_{-\sigma} .
$$

Последнее свойство интересно тем, что если встречается конструкция вида $\Pi_{\sigma} \gamma^{\mu} \Pi_{\sigma}$, то эффективно от $\gamma$-матрицы остается только ее продольная составляющая $\gamma_{\|}^{\mu}$, а в случае конструкции $\Pi_{-\sigma} \gamma^{\mu} \Pi_{\sigma}-$ поперечная часть $\gamma_{\perp}^{\mu}$.

Отметим также коммутативность проекционных операторов $\Pi_{\sigma}$ с матрицей $\gamma_{5}$ :

$$
\Pi_{\sigma} \gamma_{5}=\gamma_{5} \Pi_{\sigma}
$$


Широко используемой операцией является взятие шпура произведения некоторого числа $\gamma$-матриц. В случае сильного магнитного поля вычисление шпуров эффективно реализуется только в $\|$-подпространстве. Как и в обычном четырехмерном пространстве, в \|-подпространстве шпур нечетного числа $\gamma$-матриц равен нулю, а несколько первых шпуров четного числа - следующие:

$$
\begin{gathered}
\operatorname{Sp}\left\{\Pi_{\sigma}\right\}=2, \quad \operatorname{Sp}\left\{\gamma_{\|}^{\mu} \gamma_{\|}^{\nu} \Pi_{\sigma}\right\}=2 \tilde{\Lambda}^{\mu \nu}, \\
\operatorname{Sp}\left\{\gamma_{\|}^{\mu} \gamma_{\|}^{\nu} \gamma_{\|}^{\rho} \gamma_{\|}^{\tau} \Pi_{\sigma}\right\}=2\left[\tilde{\Lambda}^{\mu \nu} \tilde{\Lambda}^{\rho \tau}+\tilde{\Lambda}^{\mu \tau} \tilde{\Lambda}^{\nu \rho}-\tilde{\Lambda}^{\mu \rho} \tilde{\Lambda}^{\nu \tau}\right] \\
\operatorname{Sp}\left\{\gamma_{\|}^{\mu} \gamma_{\|}^{\nu} \gamma_{5} \Pi_{\sigma}\right\}=2 \sigma \tilde{\varphi}^{\mu \nu}, \quad \operatorname{Sp}\left\{\gamma_{\|}^{\mu} \gamma_{\|}^{\nu} \gamma_{\|}^{\rho} \gamma_{\|}^{\tau} \gamma_{5} \Pi_{\sigma}\right\}=2 \sigma\left[\tilde{\Lambda}^{\mu \nu} \tilde{\varphi}^{\rho \tau}+\tilde{\varphi}^{\mu \nu} \tilde{\Lambda}^{\rho \tau}\right] .
\end{gathered}
$$

Оказываются полезными и другие часто встречающиеся соотношения для $\gamma$-матриц в продольном подпространстве:

$$
\begin{gathered}
\gamma_{\| \mu} \gamma_{\|}^{\mu}=2, \\
\gamma_{\| \mu} \gamma_{\|}^{\nu} \gamma_{\|}^{\mu}=0, \\
\gamma_{\| \mu} \gamma_{\|}^{\nu} \gamma_{\|}^{\rho} \gamma_{\|}^{\mu}=2 \gamma_{\|}^{\rho} \gamma_{\|}^{\nu}, \\
\gamma_{\|}^{\mu} \gamma_{\|}^{\nu} \gamma_{\|}^{\rho}=\tilde{\Lambda}^{\mu \nu} \gamma_{\|}^{\rho}+\tilde{\Lambda}^{\nu \rho} \gamma_{\|}^{\mu}-\tilde{\Lambda}^{\mu \rho} \gamma_{\|}^{\nu}, \\
(\tilde{\varphi} \gamma)^{\mu} \Pi_{\sigma}=-\sigma \gamma_{\|}^{\mu} \gamma_{5} \Pi_{\sigma} .
\end{gathered}
$$

Легко показать, что свертка двух $\gamma_{\|}$-матриц, между которыми находится любое

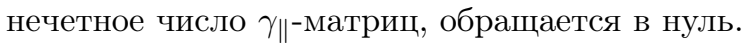

Отметим также следующее соотношение для $\gamma$-матриц в $\perp$-подпространстве:

$$
\gamma_{\perp}^{\alpha} \gamma_{\perp}^{\beta} \Pi_{\sigma}=-\left(\Lambda^{\alpha \beta}-i \sigma \varphi^{\alpha \beta}\right) \Pi_{\sigma} .
$$

Это свойство, так же как и свойство (98), позволяет эффективно снизить количество $\gamma$-матриц при вычислениях шпуров.

Отличительная особенность приведенной техники состоит в том, что она не только позволяет упростить вычисление шпуров, но и сохраняет ковариантность полученных таким способом выражений.

ПРИЛОЖЕНИЕ Б

\section{Матрица плотности заряженной частицы в импульсном пространстве}

Чтобы получить матрицу плотности заряженной спинорной частицы в постоянном однородном магнитном поле, рассмотрим вспомогательный интеграл

$$
\begin{aligned}
I_{n, p_{3}, s}^{(+)}\left(\mathrm{x}, \mathrm{x}^{\prime}\right) & =\int_{-\infty}^{\infty} d p_{2} \psi_{n, p_{2}, p_{3}, s}^{(+)}(\mathrm{x}) \bar{\psi}_{n, p_{2}, p_{3}, s}^{(+)}\left(\mathrm{x}^{\prime}\right)= \\
& =\frac{e^{-i\left[E_{n}\left(t-t^{\prime}\right)-p_{3}\left(z-z^{\prime}\right)\right]}}{2 E_{n} L_{y} L_{z}} \int_{-\infty}^{\infty} d p_{2} e^{i p_{2}\left(y-y^{\prime}\right)}\left[U_{n, p_{2}, p_{3}, s}^{(+)}(\eta) \bar{U}_{n, p_{2}, p_{3}, s}^{(+)}\left(\eta^{\prime}\right)\right],
\end{aligned}
$$

где $\eta=\sqrt{e B}\left(x-\varrho p_{2} / e B\right)$ и $\eta^{\prime}=\sqrt{e B}\left(x^{\prime}-\varrho p_{2} / e B\right)$. Перейдем от интегрирования по переменной $p_{2}$ к интегрированию по $\eta$. Выделив трансляционно-неинвариантную 
фазу $\Phi\left(\mathrm{x}, \mathrm{x}^{\prime}\right)=e B\left(x+x^{\prime}\right)\left(y-y^{\prime}\right) / 2$, получим

$$
\begin{aligned}
I_{n, p_{3}, s}^{(+)}\left(\mathrm{x}, \mathrm{x}^{\prime}\right) & =\frac{\sqrt{e B}}{2 E_{n} L_{y} L_{z}} e^{i \varrho \Phi\left(\mathrm{x}, \mathrm{x}^{\prime}\right)} e^{-i\left[E_{n}\left(t-t^{\prime}\right)-p_{3}\left(z-z^{\prime}\right)\right]} \widetilde{F}\left(\xi_{1}, \xi_{2}\right), \\
\widetilde{F}\left(\xi_{1}, \xi_{2}\right) & =e^{i \varrho \xi_{1} \xi_{2} / 2} \int_{-\infty}^{\infty} d \eta e^{-i \varrho \xi_{2} \eta}\left[U_{n, p_{2}, p_{3}, s}^{(+)}(\eta) \bar{U}_{n, p_{2}, p_{3}, s}^{(+)}\left(\eta-\xi_{1}\right)\right],
\end{aligned}
$$

где $\xi_{1}=\sqrt{e B}\left(x-x^{\prime}\right)$ и $\xi_{2}=\sqrt{e B}\left(y-y^{\prime}\right)$. Прямое и обратное фурье-преобразования функции $\widetilde{F}\left(\xi_{1}, \xi_{2}\right)$ удобно выбрать в виде

$$
\begin{aligned}
& F\left(p_{1}, p_{2}\right)=\frac{1}{(2 \pi)^{2}} \int d \xi_{1} d \xi_{2} e^{-i\left(\xi_{1} p_{1}+\xi_{2} p_{2}\right) / \sqrt{e B}} \widetilde{F}\left(\xi_{1}, \xi_{2}\right) \\
& \widetilde{F}\left(\xi_{1}, \xi_{2}\right)=\int \frac{d p_{1} d p_{2}}{e B} e^{i\left(p_{1} \xi_{1}+p_{2} \xi_{2}\right) / \sqrt{e B}} F\left(p_{1}, p_{2}\right) .
\end{aligned}
$$

Запишем интеграл (102) через фурье-образ $F\left(p_{1}, p_{2}\right)$ функции $\widetilde{F}\left(\xi_{1}, \xi_{2}\right)$ :

$$
I_{n, p_{3}, s}^{(+)}\left(\mathrm{x}, \mathrm{x}^{\prime}\right)=\frac{e^{i \varrho \Phi\left(\mathrm{x}, \mathrm{x}^{\prime}\right)}}{2 E_{n} L_{y} L_{z}} \int \frac{d p_{1} d p_{2}}{\sqrt{e B}} e^{-i p\left(\mathrm{x}-\mathrm{x}^{\prime}\right)} F\left(p_{1}, p_{2}\right) .
$$

В интеграле $I_{n, p_{3}, s}^{(+)}\left(\mathrm{x}, \mathrm{x}^{\prime}\right)$ образовался фазовый множитель $e^{-i p\left(\mathrm{x}-\mathrm{x}^{\prime}\right)}$, содержащий 4-вектор $p^{\mu}=\left(E_{n}, p_{1}, p_{2}, p_{3}\right)$, который может интерпретироваться как 4-импульс частицы. Сравнивая формулы (101) и (106), получим

$$
\psi_{n, p_{2}, p_{3}, s}^{(+)}(\mathrm{x}) \bar{\psi}_{n, p_{2}, p_{3}, s}^{(+)}\left(\mathrm{x}^{\prime}\right)=\frac{e^{i \varrho \Phi\left(\mathrm{x}, \mathrm{x}^{\prime}\right)}}{2 E_{n} L_{y} L_{z}} \int_{-\infty}^{\infty} \frac{d p_{1}}{\sqrt{e B}} e^{-i p\left(\mathrm{x}-\mathrm{x}^{\prime}\right)} F\left(p_{1}, p_{2}\right),
$$

где подынтегральная функция $F\left(p_{1}, p_{2}\right)$ может быть представлена в виде

$$
F\left(p_{1}, p_{2}\right)=e^{-2 i \varrho p_{1} p_{2} / e B} \int_{-\infty}^{\infty} \frac{d \eta}{\pi} e^{-2 i p_{1} \eta / \sqrt{e B}}\left[U_{n, p_{2}, p_{3}, s}^{(+)}(\eta) \bar{U}_{n, p_{2}, p_{3}, s}^{(+)}\left(-\eta-2 \varrho p_{2} / \sqrt{e B}\right)\right] .
$$

Вычислим $F\left(p_{1}, p_{2}\right)$ отдельно для каждой из поляризаций $s= \pm 1$, используя явный вид $U_{n, p_{2}, p_{3}, s}^{(+)}(\eta)$ (см. выражения $\left.(15)-(18)\right)$ :

$$
\begin{aligned}
F_{+\varrho}\left(p_{1}, p_{2}\right)=F & \left.\left(p_{1}, p_{2}\right)\right|_{s=\varrho}=(-1)^{n} \frac{e^{-i a b / 2}}{\pi} \int_{-\infty}^{\infty} d \eta e^{-i a \eta} \times \\
& \times\left\{\left[W_{\varrho} \bar{W}_{\varrho}\right] \chi_{n}(\eta) \chi_{n}(\eta+b)-\left[V_{-\varrho} \bar{V}_{-\varrho}\right] \chi_{n-1}(\eta) \chi_{n-1}(\eta+b)+\right. \\
& \left.+\left[W_{\varrho} \bar{V}_{-\varrho}\right] \chi_{n}(\eta) \chi_{n-1}(\eta+b)-\left[V_{-\varrho} \bar{W}_{\varrho}\right] \chi_{n-1}(\eta) \chi_{n}(\eta+b)\right\}, \\
F_{-\varrho}\left(p_{1}, p_{2}\right)=F & \left.\left(p_{1}, p_{2}\right)\right|_{s=-\varrho}=(-1)^{n} \frac{e^{-i a b / 2}}{\pi} \int_{-\infty}^{\infty} d \eta e^{-i a \eta} \times \\
& \times\left\{\left[V_{\varrho} \bar{V}_{\varrho}\right] \chi_{n}(\eta) \chi_{n}(\eta+b)-\left[W_{-\varrho} \bar{W}_{-\varrho}\right] \chi_{n-1}(\eta) \chi_{n-1}(\eta+b)+\right. \\
& \left.+\left[W_{-\varrho} \bar{V}_{\varrho}\right] \chi_{n-1}(\eta) \chi_{n}(\eta+b)-\left[V_{\varrho} \bar{W}_{-\varrho}\right] \chi_{n}(\eta) \chi_{n-1}(\eta+b)\right\},
\end{aligned}
$$


где $a=2 p_{1} / \sqrt{e B}, b=2 \varrho p_{2} / \sqrt{e B}$ и использовано свойство $\chi_{n}(-\eta)=(-1)^{n} \chi_{n}(\eta)$. Интегралы (109) и (110) берутся аналитически с помощью известного интеграла [42]

$$
\int_{-\infty}^{\infty} e^{-x^{2}} H_{m}(x+a) H_{n}(x+b) d x=2^{n} \sqrt{\pi} m ! b^{n-m} L_{m}^{n-m}(-2 a b),
$$

где $n \geqslant m$ и $L_{m}^{n-m}(x)$ - обобщенный полином Лагерра:

$$
L_{n}^{k}(x)=\frac{x^{-k} e^{x}}{n !} \frac{d^{n}}{d x^{n}}\left[x^{n+k} e^{-x}\right]
$$

Используя формулу (111), получим

$$
\int_{-\infty}^{\infty} d \eta e^{-i a \eta} \chi_{n}(\eta) \chi_{m}(\eta+b)=(-1)^{n-m} \sqrt{\frac{e B 2^{m-n} m !}{n !}}(b+i a)^{n-m} e^{\left(i a b-c^{2}\right) / 2} L_{m}^{n-m}\left(c^{2}\right),
$$

где $c^{2}=\left(a^{2}+b^{2}\right) / 2$. Отметим, что в выражения (109) и (110) функции $\chi_{n}(\eta)$ входят с индексами, различающимися не более чем на единицу. Приведем ниже требуемые интегралы:

$$
\begin{aligned}
& \int_{-\infty}^{\infty} d \eta e^{-i a \eta} \chi_{n}(\eta) \chi_{n}(\eta+b)=e^{\left(i a b-c^{2}\right) / 2} \sqrt{e B} L_{n}\left(c^{2}\right), \\
& \int_{-\infty}^{\infty} d \eta e^{-i a \eta} \chi_{n}(\eta) \chi_{n-1}(\eta+b)=e^{\left(i a b-c^{2}\right) / 2} \sqrt{e B n} \frac{i a+b}{\sqrt{2} c^{2}}\left[L_{n}\left(c^{2}\right)-L_{n-1}\left(c^{2}\right)\right], \\
& \int_{-\infty}^{\infty} d \eta e^{-i a \eta} \chi_{n}(\eta+b) \chi_{n-1}(\eta)=e^{\left(i a b-c^{2}\right) / 2} \sqrt{e B n} \frac{i a-b}{\sqrt{2} c^{2}}\left[L_{n}\left(c^{2}\right)-L_{n-1}\left(c^{2}\right)\right],
\end{aligned}
$$

где $L_{n}(x) \equiv L_{n}^{0}(x)$ - полином Лагерра, а также учтено свойство

$$
L_{n-1}^{1}(x)=\frac{n}{x}\left[L_{n-1}(x)-L_{n}(x)\right] .
$$

После интегрирования по переменной $\eta$ фурье-образ матрицы плотности определенной поляризации может быть представлен в виде

$$
\begin{gathered}
F_{+\varrho}\left(p_{1}, p_{2}\right)=(-1)^{n} \frac{\sqrt{e B}}{\pi} e^{-u / 2}\left\{L_{n}(u)\left[W_{\varrho} \bar{W}_{\varrho}\right]-L_{n-1}(u)\left[V_{-\varrho} \bar{V}_{-\varrho}\right]+\right. \\
\left.+\frac{2}{\sqrt{2 e B n}} L_{n-1}^{1}(u)\left[-\left(i p_{1}+\varrho p_{2}\right)\left[W_{\varrho} \bar{V}_{-\varrho}\right]+\left(i p_{1}-\varrho p_{2}\right)\left[V_{-\varrho} \bar{W}_{\varrho}\right]\right]\right\}, \\
F_{-\varrho}\left(p_{1}, p_{2}\right)=(-1)^{n} \frac{\sqrt{e B}}{\pi} e^{-u / 2}\left\{L_{n}(u)\left[V_{\varrho} \bar{V}_{\varrho}\right]-L_{n-1}(u)\left[W_{-\varrho} \bar{W}_{-\varrho}\right]+\right. \\
\left.+\frac{2}{\sqrt{2 e B n}} L_{n-1}^{1}(u)\left[-\left(i p_{1}-\varrho p_{2}\right)\left[W_{-\varrho} \bar{V}_{\varrho}\right]+\left(i p_{1}+\varrho p_{2}\right)\left[V_{\varrho} \bar{W}_{-\varrho}\right]\right]\right\},
\end{gathered}
$$


где $u=2\left(p_{1}^{2}+p_{2}^{2}\right) / e B$. Вычисление билинейных комбинаций биспиноров $W_{ \pm \varrho}$ и $V_{ \pm \varrho}$ с использованием их явного вида (17) и (18) приводит к следующему результату:

$$
\begin{gathered}
W_{ \pm \varrho} \bar{W}_{ \pm \varrho}=\frac{1}{2}\left[\left(1+\frac{m}{\sqrt{2 e B n+m^{2}}}\right)\left(\hat{p}_{\|}+m\right)+\frac{2 e B n}{\sqrt{2 e B n+m^{2}}}\right] \Pi_{ \pm \varrho}, \\
V_{ \pm \varrho} \bar{V}_{ \pm \varrho}=\frac{1}{2}\left[\left(1-\frac{m}{\sqrt{2 e B n+m^{2}}}\right)\left(\hat{p}_{\|}+m\right)-\frac{2 e B n}{\sqrt{2 e B n+m^{2}}}\right] \Pi_{ \pm \varrho}, \\
\frac{2}{\sqrt{2 e B n}}\left[-\left(i p_{1}+\varrho p_{2}\right)\left[W_{\varrho} \bar{V}_{-\varrho}\right]+\left(i p_{1}-\varrho p_{2}\right)\left[V_{-\varrho} \bar{W}_{\varrho}\right]\right]=\hat{p}_{\perp}-i \varrho \frac{\hat{p}_{\|}(p \varphi \gamma)}{\sqrt{2 e B n+m^{2}}}, \\
\frac{2}{\sqrt{2 e B n}}\left[-\left(i p_{1}-\varrho p_{2}\right)\left[W_{-\varrho} \bar{V}_{\varrho}\right]+\left(i p_{1}+\varrho p_{2}\right)\left[V_{\varrho} \bar{W}_{-\varrho}\right]\right]=\hat{p}_{\perp}+i \varrho \frac{\hat{p}_{\|}(p \varphi \gamma)}{\sqrt{2 e B n+m^{2}}} .
\end{gathered}
$$

Подставляя формулы (116) и (117) в (107) и сравнивая это выражение с определением (20), получим формулы (22) и (23) для матрицы плотности в импульсном пространстве.

\section{ПРИЛОЖЕНИЕ В}

\section{Вычисление интегралов по поперечным компонентам импульсов}

При вычислении импульса (10) встречаются следующие типы интегралов по импульсам заряженных частиц в поперечном подпространстве:

$$
\begin{aligned}
S^{(n, m)}(\mathrm{z}) & =\int d^{2} \mathrm{x} d^{2} \mathrm{y} \delta^{(2)}(\mathrm{x}+\mathrm{y}-\mathrm{z}) L_{n}\left(x^{2}\right) L_{m}\left(y^{2}\right) e^{-\left(x^{2}+y^{2}\right) / 2} \\
V_{\alpha}^{(n, m)}(\mathrm{z}) & =\int d^{2} \mathrm{x} d^{2} \mathrm{y} \delta^{(2)}(\mathrm{x}+\mathrm{y}-\mathrm{z}) x_{\alpha} L_{n-1}^{1}\left(x^{2}\right) L_{m}\left(y^{2}\right) e^{-\left(x^{2}+y^{2}\right) / 2} \\
T_{\alpha \beta}^{(n, m)}(\mathrm{z}) & =\int d^{2} \mathrm{x} d^{2} \mathrm{y} \delta^{(2)}(\mathrm{x}+\mathrm{y}-\mathrm{z}) x_{\alpha} y_{\beta} L_{n-1}^{1}\left(x^{2}\right) L_{m-1}^{1}\left(y^{2}\right) e^{-\left(x^{2}+y^{2}\right) / 2},
\end{aligned}
$$

где $\mathrm{x}, \mathrm{y}$ и $\mathrm{z}-$ векторы в двумерном (поперечном) пространстве $(\alpha, \beta=1,2), \mathrm{a} \delta^{(2)}(\mathrm{x}+$ $\mathrm{y}-\mathrm{z})=\delta\left(x_{1}+y_{1}-z_{1}\right) \delta\left(x_{2}+y_{2}-z_{2}\right)-$ произведение $\delta$-функций Дирака.

При использовании фурье-образа $\delta$-функции

$$
\delta^{(2)}(\mathrm{x}+\mathrm{y}-\mathrm{z})=\frac{1}{(2 \pi)^{2}} \int d^{2} \mathrm{~s} e^{i \mathrm{~s}(\mathrm{x}+\mathrm{y}-\mathrm{z})}
$$

где $\mathrm{sx}=s_{1} x_{1}+s_{2} x_{2}-$ скалярное произведение векторов в двумерном евклидовом пространстве, интегралы по х и у становятся независимым и принимают вид

$$
\begin{aligned}
& f^{(n)}(\mathrm{s})=\int d^{2} \mathrm{x} L_{n}\left(x^{2}\right) e^{i(\mathrm{sx})-x^{2} / 2} \\
& f_{\alpha}^{(n)}(\mathrm{s})=\int d^{2} \mathrm{x} x_{\alpha} L_{n-1}^{1}\left(x^{2}\right) e^{i(\mathrm{sx})-x^{2} / 2} .
\end{aligned}
$$

Векторный интеграл (122) пропорционален вектору $\mathrm{s}_{\alpha}$ :

$$
f_{\alpha}^{(n)}(\mathrm{s})=\mathrm{s}_{\alpha} A^{(n)}\left(\mathrm{s}^{2}\right)
$$


а функция $A^{(n)}\left(\mathrm{s}^{2}\right)$ находится из свертки $\mathrm{s}_{\alpha} f_{\alpha}^{(n)}(\mathrm{s})$. Получившиеся скалярные интегралы $f^{(n)}(\mathrm{s})$ и $A^{(n)}\left(\mathrm{s}^{2}\right)$ удобно вычислять в полярных координатах, отсчитывая полярный угол от вектора $\mathrm{s}$. Воспользовавшись известными соотношениями [42]

$$
\begin{aligned}
\int_{0}^{2 \pi} e^{ \pm i t \cos \varphi} \cos (n \varphi) d \varphi & =( \pm i)^{n} 2 \pi J_{n}(t) \\
\int_{0}^{\infty} t^{\lambda / 2} e^{-c t / 2} J_{\lambda}(b \sqrt{t}) L_{n}^{\lambda}(t) d t & =(-1)^{n} 2 b^{\lambda} c^{-\lambda-1} e^{-b^{2} / 2 c} L_{n}^{\lambda}\left(\frac{b^{2}}{c}\right)
\end{aligned}
$$

где $J_{n}(t)$ - функция Бесселя первого рода, получим следующие выражения для интегралов (121) и (122):

$$
\begin{aligned}
& f^{(n)}(\mathrm{s})=(-1)^{n} 2 \pi e^{-\mathrm{s}^{2} / 2} L_{n}\left(\mathrm{~s}^{2}\right), \\
& f_{\alpha}^{(n)}(\mathrm{s})=i(-1)^{n-1} 2 \pi \mathrm{s}_{\alpha} e^{-\mathrm{s}^{2} / 2} L_{n-1}^{1}\left(\mathrm{~s}^{2}\right) .
\end{aligned}
$$

В терминах функций $f^{(n)}(\mathrm{s})$ и $f_{\alpha}^{(n)}(\mathrm{s})$ интегралы (118)-(120) представляются в виде

$$
\begin{aligned}
S^{(n, m)}(\mathrm{z}) & =\int \frac{d^{2} \mathrm{~s}}{(2 \pi)^{2}} e^{-i(\mathrm{sz})} f^{(n)}(\mathrm{s}) f^{(m)}(\mathrm{s}), \\
V_{\alpha}^{(n, m)}(\mathrm{z}) & =\int \frac{d^{2} \mathrm{~s}}{(2 \pi)^{2}} e^{-i(\mathrm{sz})} f_{\alpha}^{(n)}(\mathrm{s}) f^{(m)}(\mathrm{s}), \\
T_{\alpha \beta}^{(n, m)}(\mathrm{z}) & =\int \frac{d^{2} \mathrm{~s}}{(2 \pi)^{2}} e^{-i(\mathrm{sz})} f_{\alpha}^{(n)}(\mathrm{s}) f_{\beta}^{(m)}(\mathrm{s}) .
\end{aligned}
$$

Векторная и тензорная структуры интегралов $V_{\alpha}^{(n, m)}(\mathrm{z})$ и $T_{\alpha \beta}^{(n, m)}(\mathrm{z})$ очевидны:

$$
\begin{gathered}
V_{\alpha}^{(n, m)}(\mathrm{z})=B^{(n, m)} \mathrm{z}_{\alpha}, \\
T_{\alpha \beta}^{(n, m)}(\mathrm{z})=C^{(n, m)} \delta_{\alpha \beta}+D^{(n, m)} \mathrm{z}_{\alpha} \mathrm{z}_{\beta} .
\end{gathered}
$$

Скалярные интегралы $B^{(n, m)}, C^{(n, m)}$ и $D^{(n, m)}$ также удобно вычислять в полярных координатах, отсчитывая угол от вектора z. Используя соотношение (123) и формулу [42]

$$
\begin{aligned}
& \int_{0}^{\infty} d t t^{(\varkappa+\lambda) / 2} e^{-c t} J_{\varkappa+\lambda}(b \sqrt{t}) L_{p}^{\varkappa}(t) L_{k}^{\lambda}(t)= \\
& \quad=\frac{(-1)^{p+k}}{c}\left(\frac{b}{2 c}\right)^{\varkappa+\lambda} e^{-b^{2} / 4} L_{p}^{\lambda+k-p}\left(\frac{b^{2}}{4 c}\right) L_{k}^{\varkappa+p-k}\left(\frac{b^{2}}{4 c}\right),
\end{aligned}
$$

мы можем представить интегралы $S^{(n, m)}(\mathrm{z}), V_{\alpha}^{(n, m)}(\mathrm{z})$ и $T_{\alpha \beta}^{(n, m)}(\mathrm{z})$ в виде

$$
\begin{aligned}
S^{(n, m)}(\mathrm{z}) & =\pi e^{-z^{2} / 4} L_{n}^{m-n}\left(\frac{z^{2}}{4}\right) L_{m}^{n-m}\left(\frac{z^{2}}{4}\right), \\
2 V_{\alpha}^{(n, m)}(\mathrm{z})= & \pi e^{-z^{2} / 4} z_{\alpha} L_{n-1}^{m-n+1}\left(\frac{z^{2}}{4}\right) L_{m}^{n-m}\left(\frac{z^{2}}{4}\right), \\
8 T_{\alpha \beta}^{(n, m)}(\mathrm{z})= & \pi e^{-z^{2} / 4}\left[\left(2 z_{\alpha} z_{\beta}-z^{2} \Lambda_{\alpha \beta}\right) L_{n-1}^{m-n+1}\left(\frac{z^{2}}{4}\right) L_{m-1}^{n-m+1}\left(\frac{z^{2}}{4}\right)-\right. \\
& \left.-4 n \Lambda_{\alpha \beta} L_{n}^{m-n}\left(\frac{z^{2}}{4}\right) L_{m-1}^{n-m}\left(\frac{z^{2}}{4}\right)\right] .
\end{aligned}
$$


В тензорном интеграле символ Кронекера $\delta_{\alpha \beta}$ заменен эквивалентным метрическим тензором $\Lambda_{\alpha \beta}$ двумерного поперечного евклидова подпространства.

Благодарности. Авторы выражают искреннюю признательность И. С. Огневу за сотрудничество на начальном этапе работы. Авторы благодарны Н. В. Михееву и Д. А. Румянцеву за плодотворные обсуждения, а также А. В. Борисову и А. И. Студеникину за ценные замечания. Исследование проведено в рамках реализации ФЦП "Научные и научно-педагогические кадры инновационной России" на 2009-2013 гг. (госконтракт № П2323) при частичной финансовой поддержке Министерства образования и науки РФ по программе "Развитие научного потенциала высшей школы" (проект№ 2.1.1/13011) и РФФИ (грант № 11-02-00394-а).

\section{Список литературы}

[1] Г. С. Бисноватый-Коган, Астроном. ж., 47 (1970), 813-816.

[2] N. V. Ardeljan, G. S. Bisnovatyi-Kogan, S. G. Moiseenko, Mon. Not. Roy. Astron. Soc., 359:1 (2005), 333-344, arXiv: astro-ph/0410234.

[3] Н. Н. Чугай, Писъма в Астроном. ж., 10:2 (1984), 210-213.

[4] О. Ф. Дорофеев, В. Н. Родионов, И. М. Тернов, Писъма в ЖЭТФ, 40:4 (1984), 159-161.

[5] О.Ф. Дорофеев, В.Н. Родионов, И. М. Тернов, Писъма в Астроном. ж., 11 (1985), 302-309.

[6] Ю. М. Лоскутов, Писъма в ЖЭТФ, 39:9 (1984), 438-440; ТМФ, 65:1 (1985), 141-150.

[7] В. М. Захарцов, Ю. М. Лоскутов, Вестник МГУ. Сер. 3. Физ. Астрон., 26:2 (1985), $24-30$.

[8] А. И. Студеникин, Астрофизика, 28:3 (1988), 638-647.

[9] Ю. М. Лоскутов, Ю. М. Парфенов, Вестник МГУ. Сер. 3. Физ. Астрон., 30 (1989), $3-7$.

[10] А. А. Гвоздев, И. С. Огнев, Писъма в ЖКЭТФ, 69:5 (1999), 337-342.

[11] А. А. Гвоздев, И. С. Огнев, ЖКЭТФ, 121:6 (2002), 1219-1234.

[12] S. Mereghetti, Astron. Astrophys. Rev., 15:4 (2008), 225-287.

[13] R. C. Duncan, C. Thompson, Astrophys. J., 392:1 (1992), L9-L13.

[14] C. Thompson, R. C. Duncan, Astrophys. J., 408:1 (1993), 194-217.

[15] C. Thompson, R. C. Duncan, Astrophys. J., 473:1 (1996), 322-342.

[16] C. Thompson, R. C. Duncan, Mon. Not. Roy. Astron. Soc., 275:2 (1995), 255-300.

[17] А.А. Гвоздев, И.С. Огнев, Е.В. Осокина, Писъма в Астроном. ж., 37:5 (2011), 365-376, arXiv: 1105.4080.

[18] H. A. Bethe, Rev. Mod. Phys., 62:4 (1990), 801-866.

[19] N. Itoh, H. Hayashi, A. Nishikava, Y. Kohyama, Astrophys. J. Suppl. Ser., 102 (1996), 411-424.

[20] Л. И. Коровина, Известия вузов. Физика, 6 (1964), 86-92.

[21] И. М. Тернов, Б. А. Лысов, Л. И. Коровина, Вестник МГУ. Сер. 3. Физ. Астрон., 6:5 (1965), 58-63.

[22] J. J. Matese, R.F. O'Connell, Phys. Rev., 180:5 (1969), 1289-1292.

[23] L. Fassio-Canuto, Phys. Rev., 187:5 (1969), 2141-2146.

[24] S. Shinkevich, A. Studenikin, Pramana, 65:2 (2005), 215-244, arXiv: hep-ph/0402154.

[25] А. И. Студеникин, ЯФ, 49 (1989), 1665-1671.

[26] D. G. Yakovlev, A. D. Kaminker, O. Y. Gnedin, P. Haensel, Phys. Rept., 354:1-2 (2001), 1-155, arXiv: astro-ph/0012122.

[27] K. Bhattacharya, P. B. Pal, Proc. Ind. Natl. Sci. Acad., 70 (2004), 145-161, arXiv: hep-ph/0212118. 
[28] A. V. Kuznetsov, N. V. Mikheev, Electroweek Processes in External Electromagnetic Fields, Springer Tracts in Modern Physics, 197, Springer, New York, 2004.

[29] E. Storck, Z. Natur. Forsch., 23 (1968), 1914-1928.

[30] М. С. Андреев, Н. В. Михеев, Е. Н. Нарынская, ЖЖЭТФ, 137:2 (2010), 259-267.

[31] А. А. Соколов, И. М. Тернов, Релятивистский электрон, Наука, М., 1974.

[32] Е. Янке, Ф. Эмде, Ф. Леш, Специалъные функиии. Формуль, графики, таблицы, Наука, М., 1977.

[33] K. Nakamura et. al. (Particle Data Group), J. Phys. G, 37:7A (2010), 075021, 1422 pp.

[34] G. G. Raffelt, Stars as Laboratories for Fundamental Physics, Univ. Chicago Press, Chicago, 1996.

[35] J. D. Landstreet, Phys. Rev., 153:5 (1967), 1372-1377.

[36] V. G. Bezchastnov, P. Haensel, A. D. Kaminker, D. G. Yakovlev, Astron. Astrophys., 328 (1997), 409-418.

[37] А. Д. Каминкер, Д. Г. Яковлев, ТМФ, 49:2 (1981), 248-260.

[38] A. D. Kaminker, O. Yu. Gnedin, D. G. Yakovlev, Phys. Rev. D, 46:10 (1992), 4133-4139.

[39] А. Д. Каминкер, Д. Г. Яковлев, ЖКЭТФ, 2 (1993), 438-454.

[40] В. В. Скобелев, Известия вузов. Физика, 10 (1975), 142-143.

[41] Yu. M. Loskutov, V. V. Skobelev, Phys. Lett. A, 56:3 (1976), 151-152.

[42] А. П. Прудников, Ю. А. Брычков, О. И. Маричев, Интегралы и рлды, т. 2: Специальные функции, Физматлит, М., 2003.

Поступила в редакцию 6.05.2011 